\title{
MATROIDS AND GRAPHS
}

BY

W. T. TUTTE

1. Introduction. This paper is a sequel to two others, [3] and [4], recently published in these Transactions. It also uses the definitions and theorems of [2]. We refer to these three papers as HI, HII and A respectively.

In the original paper on matroids, [6], Hassler Whitney pointed out that the circuits of any finite graph $G$ define a matroid. We call this the circuitmatroid and its dual the bond-matroid of $G$. In the present paper we determine a necessary and sufficient condition, in terms of matroid structure, for a given matroid $M$ to be graphic (cographic), that is the bond-matroid (circuitmatroid) of some finite graph. The condition is that $\boldsymbol{M}$ shall be regular and shall not contain, in a sense to be explained, the circuit-matroid (bondmatroid) of a Kuratowski graph, that is a graph with one of the structures shown in Figure I.

Some of the intermediate results seem to be of interest in themselves. These include the theory of dual matroids in $\$ 2$, Theorem (7.3) on regular matroids, and Theorem (8.4) on the bond-matroids of graphs.

Our main theorem is evidently closely related to the theorem of Kuratowski on planar graphs [1]. This states that a graph is planar (i.e., can be imbedded in the plane) if and only if it contains no graph with the point set structure of a Kuratowski graph. Indeed it is not difficult to prove Kuratowski's Theorem from ours, using the principle that a graph is planar if and only if it has a dual graph, that is if and only if its circuit-matroid is graphic. However this paper is long enough already and we refrain from adding matter not essential to the proof and understanding of the main theorem.

2. Dual matroids. We define a matroid $\boldsymbol{M}$ on a set $M$, its flats and their dimensions as in $\mathrm{HI}, \S 1$. We call the dimension of the largest flat $\langle M\rangle$ also the dimension $d M$ of the matroid. (See HI, $\S 2$, for the notation $\langle S\rangle$ ). We write $\alpha(S)$ for the number of elements of any finite set $S$. We proceed to give a definition of the dual of $M$ analogous to the definition of a dual vector space in terms of orthogonality.

First, in analogy with $A, \S 4$, we define a dendroid of $M$ as a minimal subset $D$ of $M$ which meets every $X \in M$. Then for each $a \in D$ there is a point $J(\boldsymbol{M}, D, a)$ of $\boldsymbol{M}$ such that $D \cap J(\boldsymbol{M}, D, a)=\{a\}$. Moreover $J(\boldsymbol{M}, D, a)$ is unique, for if $(X, Y) \in M, X \cap D=Y \cap D=\{a\}$ and $X \neq Y$ it follows by Axiom II of the definition of a matroid that there exists $Z \in M$ such that $Z \subseteq(X \cup Y)-\{a\} \subseteq M-D$.

(2.1) If $a \in X \in M$ there exists a dendroid $D$ of $\boldsymbol{M}$ such that $X=J(M, D, a)$.

Received by the editors April 27, 1957. 
Proof. By Axiom I $(M-X) \cup\{a\}$ meets every $Y \in M$. It thus has a dendroid $D$ of $\boldsymbol{M}$ as a subset. But $a \in D$ since $D \cap X \neq \varnothing$. Hence $X=J(\boldsymbol{M}, D, a)$.

(2.2) If $D$ is a dendroid of $M$ then $\alpha(D)=d M+1$.

Proof. If $D=\varnothing$, then $\boldsymbol{M}$ is a null class and $d \boldsymbol{M}=-1$. In the remaining case we enumerate the cells of $D$ as $a_{1}, \cdots, a_{k}$ and write $S_{0}=\langle M\rangle, S_{i}$ $=\left\langle M-\left\{a_{1}, \cdots, a_{i}\right\}\right\rangle(i=1, \cdots, k)$. It is clear that $J\left(M, D, a_{i}\right) \subseteq S_{i-1}$ and so $a_{i} \in S_{i-1}$ and $S_{i}=\left\langle S_{i-1}-\left\{a_{i}\right\}\right\rangle$. But $d S_{k}=-1$ since $D \cap S_{k}=\varnothing$. Hence $d M=d S_{0}=\alpha(D)-1$, by HI, (2.3).

(2.3) If $\boldsymbol{M}^{\prime}$ is a matroid on $M$ having the same dendroids as $\boldsymbol{M}$, then $M^{\prime}=M$.

Proof. Let $D$ be any dendroid of $\boldsymbol{M}$ and $\boldsymbol{M}^{\prime}$, and $a$ any cell of $D$. Suppose $a \neq b \in J(\boldsymbol{M}, D, a)$. Then $(D-\{a\}) \cup\{b\}$ meets every $X \in M$ and so contains a dendroid $E$ of $\boldsymbol{M}$. Clearly $b \in E$. Since $J\left(\boldsymbol{M}^{\prime}, D, a\right)$ meets $E$ we have $b \in J\left(\boldsymbol{M}^{\prime}, D, a\right)$. We deduce that $J(\boldsymbol{M}, D, a) \subseteq J\left(\boldsymbol{M}^{\prime}, D, a\right)$. Similarly $J\left(\boldsymbol{M}^{\prime}, D, a\right) \subseteq J(\boldsymbol{M}, D, a)$ and therefore $J(\boldsymbol{M}, D, a)=J\left(\boldsymbol{M}^{\prime}, D, a\right)$. The theorem follows by (2.1).

We call two subsets $S$ and $T$ of $M$ orthogonal if $\alpha(S \cap T) \neq 1$. We write $L(M)$ for the class of all non-null subsets $S$ of $M$ such that $S$ is orthogonal to every $X \in M$, and $M^{*}$ for the class of all minimal members of $L(M)$.

(2.4) If $(X, Y) \in L(M), a \in X \cap Y$ and $b \in X-(X \cap Y)$, then there exists $Z \in L(\boldsymbol{M})$ such that $b \in Z \subseteq(X \cup Y)-\{a\}$.

Proof. Assume the theorem false for some $X, Y, a, b$. We construct a sequence $\left(a_{0}, a_{1}, \cdots, a_{k}\right)$ of cells of $X \cup Y$ and a sequence $\left(T_{1}, \cdots, T_{k}\right)$ of points of $\boldsymbol{M}$ as follows. We put $a_{0}=a$. If we have determined the cells $a_{i}$ as far as $a_{r}$, and $b$ is not among them, we take $T_{r+1}$ to be any point of $M$ such that $T_{r+1} \cap\left((X \cup Y)-\left\{a_{0}, \cdots, a_{r}\right\}\right)$ has just one cell, $c$ say. This is possible since $(X \cup Y)-\left\{a_{0}, \cdots, a_{r}\right\} \in L(M)$, by our assumption. We then write $a_{r+1}=c$. This construction terminates with the first $a_{k}$ identical with $b$.

There exists $U \in M$ such that $b \in U \cap(X \cup Y) \subseteq\left\{a_{0}, \cdots, a_{k}\right\}$, for example $U=T_{k}$. For each such $U$ let $p(U)$ be the greatest $j<k$ such that $a_{j} \in U$. Such a $j$ exists since $\alpha(U \cap X) \neq 1$. If $p(U)>0$ we can apply Axiom II to $U$ and $T_{p(U)}$ to prove the existence of $U^{\prime} \in M$ such that $b \in U^{\prime} \cap(X \cup Y)$ $\subseteq\left\{a_{0}, \cdots, a_{k}\right\}$ and $p\left(U^{\prime}\right)<p(U)$. Hence we can choose $U$ so that $p(U)=0$. But then $Y \notin L(\boldsymbol{M})$.

(2.5) $M^{*}$ is a matroid on $M$.

Proof. By its definition $\boldsymbol{M}^{*}$ satisfies Axiom I. Suppose $(X, Y) \in \boldsymbol{M}^{*}$, $a \in X \cap Y$ and $b \in X-(X \cap Y)$. By (2.4) there exists $Z \in L(M)$ such that $b \in Z \subseteq(X \cup Y)-\{a\}$. Choose such a $Z$ so that $\alpha(Z)$ is as small as possible. If $Z \notin M^{*}$ there exists $T \in L(M)$ such that $T \subseteq Z-\{b\}$. Choose $c \in T$. By (2.4) there exists $Z^{\prime} \in L(M)$ such that $b \in Z^{\prime} \subseteq\left(Z \cup T^{\prime}\right)-\{c\}$, which contradicts the definition of $Z$. Hence $Z \in M^{*}$. Thus $M^{*}$ satisfies Axiom II.

We call $\boldsymbol{M}^{*}$ the dual matroid of $\boldsymbol{M}$.

If $D$ is a dendroid of $\boldsymbol{M}$ and $b \in M-D$ we define $K(\boldsymbol{M}, D, b)$ as the subset of $M$ consisting of $b$ and each $a \in D$ such that $b \in J(\boldsymbol{M}, D, a)$. 
(2.6) $K(\boldsymbol{M}, D, b) \in \boldsymbol{M}^{*}$.

Proof. If $K(\boldsymbol{M}, D, b) \in L(\boldsymbol{M})$ choose $X \in \boldsymbol{M}$ such that $\alpha(X \cap K(\boldsymbol{M}, D, b))$ $=1$ and $\alpha(X \cap D)$ has the least value consistent with this. Write $X \cap K(\boldsymbol{M}, D, b)$ $=\{c\}$. If $e$ belongs to $D \cap X$ but not to $K(\boldsymbol{M}, D, b)$ we have $b \notin J(\boldsymbol{M}, D, e)$ and so $c \notin J(M, D, e)$. By Axiom II there exists $X^{\prime} \in M$ such that $c \in X^{\prime}$ $\subseteq(X \cup J(\boldsymbol{M}, D, e))-\{e\}$. But then $\alpha\left(X^{\prime} \cap K(\boldsymbol{M}, D, b)\right)=1$ and $\alpha\left(X^{\prime} \cap D\right)$ $<\alpha(X \cap D)$, contrary to the definition of $X$. Since $X \cap D \neq \varnothing$ we deduce that $c \in D$ and $X=J(\boldsymbol{M}, D, c)$. But $c \in K(\boldsymbol{M}, D, b)$. Hence $b \in J(\boldsymbol{M}, D, c)=X$ and $\alpha(X \cap K(\boldsymbol{M}, D, b)) \geqq 2$, contrary to the definition of $X$. We deduce that $K(\boldsymbol{M}, D, b) \in \boldsymbol{L}(\boldsymbol{M})$. As no non-null proper subset of $K(\boldsymbol{M}, D, b)$ is orthogonal to all the sets $J(\boldsymbol{M}, D, a)$ it follows that $K(\boldsymbol{M}, D, b) \in \boldsymbol{M}^{*}$.

(2.7) The dendroids of $\boldsymbol{M}^{*}$ are the complements in $M$ of the dendroids of $\boldsymbol{M}$.

Proof. Let $D$ be any dendroid of $\boldsymbol{M}$. Then $M-D$ meets each $Y \in \boldsymbol{M}^{*}$, for no non-null subset of $D$ is orthogonal to all the sets $J(\boldsymbol{M}, D, a)$. But no proper subset of $M-D$ meets all the sets $K(M, D, b)$. Hence $M-D$ is a dendroid of $M^{*}$, by (2.6).

Conversely suppose $D^{\prime}$ is a dendroid of $\boldsymbol{M}^{*}$. Then $M-D^{\prime}$ meets each $X \in M$, for no non-null subset of $D^{\prime}$ is orthogonal to all the sets $J\left(M^{*}, D^{\prime}, a\right)$. Hence $M$ has a dendroid $D$ such that $D \subseteq M-D^{\prime}$, that is $D^{\prime} \subseteq M-D$. But $M-D$ is a dendroid of $M^{*}$ by the result of the preceding paragraph. Hence $D^{\prime}=M-D$.

Corollary I. $d \boldsymbol{M}+d \boldsymbol{M}^{*}=\alpha(M)-2$

Corollary II. $K(\boldsymbol{M}, D, b)=J\left(\boldsymbol{M}^{*}, M-D, b\right)$

$$
\left(M^{*}\right)^{*}=M \text {. }
$$

Proof. By (2.7) $M$ and $\left(M^{*}\right)^{*}$ have the same dendroids. Hence they are identical, by (2.3).

For each $S \subseteq M$ we have the following identities.

$$
\begin{aligned}
& M^{*} \times S=(M \cdot S)^{*}, \\
& M^{*} \cdot S=(M \times S)^{*} .
\end{aligned}
$$

The notation is that of HII, $\S 3$. To prove (2.9) suppose $X \in M^{*} \times S$. Then $X \in M^{*}$, that is $X$ is orthogonal to each $Y \in M$. Hence $X$ is orthogonal to the intersection with $S$ of each $Y \in M$ and therefore $X \in L(M \cdot S)$. Accordingly there exists $X^{\prime} \in(\boldsymbol{M} \cdot S)^{*}$ such that $X^{\prime} \subseteq X$. Conversely suppose $X^{\prime} \in(\boldsymbol{M} \cdot S)^{*}$. Then $X^{\prime}$ is orthogonal to each member of $\boldsymbol{M} \cdot S$, and therefore to each $Y \in \boldsymbol{M}$, by HII, (3.1). Hence $X^{\prime} \in L(\boldsymbol{M})$ and there exists $X \in M^{*} \times S$ such that $X \subseteq X^{\prime}$. Formula (2.9) now follows by Axiom I. To prove (2.10) we write $\boldsymbol{M}^{*}$ for $\boldsymbol{M}$ in (2.9), take dual matroids and use (2.8).

Let $R$ denote either the ring of integers or the ring of residues modulo a prime. Let $N$ be a chain-group on $M$ over $R$, as defined in HII, $\S 1$. The dual chain-group $N^{*}$ of $N$ clearly has the property that $\alpha(|f| \cap|g|) \neq 1$ for any 
$f \in N$ and $g \in N^{*}$. Hence if $X \in \boldsymbol{M}\left(N^{*}\right)$ there exists $Y \in(\boldsymbol{M}(N))^{*}$ such that $Y \subseteq X$.

If $Y \in(\boldsymbol{M}(N))^{*}$ we can write $Y=K(\boldsymbol{M}(N), D, b)$, for suitable $D$ and $b$, by (2.1) and (2.7), Corollary II. We can find $d \boldsymbol{M}(N)+1$ linearly independent chains $f_{a}(a \in D)$ of $N$ such that $\left|f_{a}\right|=J(\boldsymbol{M}(N), D, a)$, by (2.2). We define a chain $g$ on $M$ over $R$ such that $|g|=Y$ according to the following rules.

$$
\begin{aligned}
& g(b)=-\prod_{a \in D} f_{a}(a), \\
& g(c)=f_{c}(b) \prod_{a \in D-\{c\}} f_{a}(a), \quad(c \in D) .
\end{aligned}
$$

It is easily verified that

$$
\sum_{e \in M} f(e) g(e)=0
$$

if $f$ is any of the chains $f_{a}$. Hence the relation is true for each $f \in N$, since each such chain is linearly dependent on the $f_{a}$, by HII, (2.4). Hence $g \in N^{*}$. Accordingly there exists $X \in M\left(N^{*}\right)$ such that $X \subseteq Y$.

Applying Axiom I to the above results we obtain

$$
\boldsymbol{M}\left(N^{*}\right)=(\boldsymbol{M}(N))^{*} .
$$

(2.12) The dual of a binary (regular) matroid is binary (regular).

The terminology here is that of HII, $\S 1$. The theorem is a consequence of (2.11) and A, (5.1).

3. Minors. Let $M$ be any matroid on a set $M$. Then if $T \subseteq S \subseteq M$ we have the following identities.

$$
\begin{aligned}
& (\boldsymbol{M} \times S) \times T=M \times T, \\
& (\boldsymbol{M} \cdot S) \cdot T=\boldsymbol{M} \cdot T, \\
& (\boldsymbol{M} \cdot S) \times T=(\boldsymbol{M} \times(M-(S-T))) \cdot T, \\
& (\boldsymbol{M} \times S) \cdot T=(\boldsymbol{M} \cdot(M-(S-T))) \times T .
\end{aligned}
$$

Formula (3.1) follows at once from the definition of $M \times S$ in HII, §3. To prove (3.2) we write $\boldsymbol{M}^{*}$ for $\boldsymbol{M}$ in (3.1), apply (2.9) and take dual matroids.

To prove (3.3) suppose $X \in(\boldsymbol{M} \cdot S) \times T$. Then there exists $X^{\prime} \in \boldsymbol{M}$ such that $X^{\prime} \cap(S-T)=\varnothing$ and $X^{\prime} \cap T=X$. But then $X^{\prime} \in M \times(M-(S-T))$ and there exists $Y \in(M \times(M-(S-T))) \cdot T$ such that $Y \subseteq X^{\prime} \cap T=X$. Conversely suppose $Y \in(\boldsymbol{M} \times(M-(S-T))) \cdot T$. Then there exists $Y^{\prime} \in \boldsymbol{M}$ such that $Y^{\prime} \cap(S-T)=\varnothing$ and $Y^{\prime} \cap T=Y$. Hence there exists $X \in(M \cdot S) \times T$ such that $X \subseteq Y^{\prime} \cap T=Y$. Now (3.3) follows by Axiom I.

We obtain (3.4) by writing $M-(S-T)$ for $S$ in (3.3).

We refer to the matroids of the form $(\boldsymbol{M} \times S) \cdot T$ as the minors of $\boldsymbol{M}$. By (3.3) and (3.4) they are also the matroids of the form $(\boldsymbol{M} \cdot S) \times T$. We note 
that $\boldsymbol{M}, \boldsymbol{M} \cdot \boldsymbol{S}$ and $\boldsymbol{M} \times S$ are minors of $\boldsymbol{M}$, for $\boldsymbol{M}=(\boldsymbol{M} \times M) \cdot \boldsymbol{M}, \boldsymbol{M} \cdot \boldsymbol{S}$ $=(\boldsymbol{M} \times M) \cdot S$ and $\boldsymbol{M} \times S=(\boldsymbol{M} \times S) \cdot S$.

(3.5) Every minor of a minor of $\boldsymbol{M}$ is a minor of $\boldsymbol{M}$.

(3.6) The minors of $\boldsymbol{M}^{*}$ are the dual matroids of the minors of $\boldsymbol{M}$.

(3.7) The minors of a binary (regular) matroid are binary (regular).

(3.5) is a consequence of the identities (3.1)-(3.4), and (3.6) is implied by (2.9) and (2.10). (3.7) follows from HII, (4.3).

A subset $S$ of $M$ is a separator of $M$ if no $X \in M$ meets both $S$ and $M-S$. We call $M$ connected if it has no separator other than the trivial ones $\varnothing$ and $M$. We have at once:

(3.8) If $S$ is a separator of $M$ and $U \subseteq M$, then $S \cap U$ is a separator of both $M \times U$ and $M \cdot U$.

(3.9) $A$ subset $S$ of $M$ is a separator of $\boldsymbol{M}$ if and only if $\boldsymbol{M} \times S=\boldsymbol{M} \cdot S$.

Proof. Suppose $S$ is a separator of $\boldsymbol{M}$. If $X \in \boldsymbol{M} \cdot S$ there exists $X^{\prime} \in \boldsymbol{M}$ such that $X=X^{\prime} \cap S$. Then $X^{\prime}=X$ since $S$ is a separator, and so $X \in M \times S$. Conversely suppose $X \in \boldsymbol{M} \times S$. Then $X \in \boldsymbol{M}$ and there exists $Y \in \boldsymbol{M} \cdot S$ such that $Y \subseteq X$. By the result just proved $Y \in M \times S$ and therefore $X=Y \in M \cdot S$, by Axiom I.

Next suppose $S$ is not a separator of $M$. Then there exists $X \in M$ such that $X \cap S$ and $X \cap(M-S)$ are both non-null. There exists $Y \in M \cdot S$ such that $Y \subseteq X \cap S$. But then $Y \notin M$, by Axiom I, and so $Y \notin M \times S$. Hence $M \times S \neq M \cdot S$.

We define an elementary separator of $M$ as a minimal non-null separator of $\boldsymbol{M}$. It is clear that if $M \neq \varnothing$ the elementary separators of $\boldsymbol{M}$ are disjoint and have $M$ as their union. An elementary separator may consist of a single cell $a$. This happens when either $\{a\} \in M$ or no point of $\boldsymbol{M}$ includes $a$. An elementary separator of $\boldsymbol{M}$ having more than one cell is necessarily a connected flat of $\boldsymbol{M}$, as defined in HI, $\$ 1$.

(3.10) The elementary separators of $\boldsymbol{M}^{*}$ are identical with those of $\boldsymbol{M}$.

This follows from (2.9), (2.10) and (3.9).

4. Circuits and bonds. For convenience we state here some of the fundamental definitions of graph theory which were assumed in A.

A graph $G$ is defined by a set $E(G)$ of edges, a set $V(G)$ of vertices, and a relation of incidence which associates with each edge a pair of vertices, not necessarily distinct, called its ends. An edge is a link or loop according as its ends are distinct or coincident. A vertex which is not an end of any edge is called isolated.

In this paper we suppose $E(G)$ and $V(G)$ both finite. We denote the number of members of $E(G)$ and $V(G)$ by $\alpha_{1}(G)$ and $\alpha_{0}(G)$ respectively.

A graph $H$ is a subgraph of $G$ if $E(H) \subseteq E(G), V(H) \subseteq V(G)$ and each $A \in E(H)$ has the same ends in $H$ as in $G$. The union of given subgraphs $G_{1}, \cdots, G_{k}$ of $G$ is the subgraph $H$ of $G$ such that $E(H)=\bigcup_{i} E\left(G_{i}\right)$ and $V(H)=\bigcup_{i} V\left(G_{i}\right)$. If $W \subseteq V(G)$ we write $G[W]$ for the subgraph of $G$ whose 
vertices are the members of $W$ and whose edges are those edges of $G$ which have both ends in $W$.

A sequence $P=\left(a_{0}, A_{1}, a_{1}, \cdots, A_{n}, a_{n}\right)$, having at least one term, is a path in $G$ from $a_{0}$ to $a_{n}$ if the following conditions are satisfied.

(i) The terms of $P$ are alternately vertices $a_{i}$ and edges $A_{j}$ of $G$.

(ii) If $1 \leqq j \leqq n$ then $a_{j-1}$ and $a_{j}$ are the two ends in $G$ of $A_{j}$.

We call $P$ degenerate if it has only one term, simple if the $a_{i}$ are all distinct, and re-entrant if $a_{0}=a_{n}$. We call it circular if it is re-entrant, nondegenerate and such that the vertices $a_{0}, \cdots, a_{n-1}$ are all distinct.

Clearly if there is any path in $G$ from $a_{0}$ to $a_{n}$ there is a simple path in $G$ from $a_{0}$ to $a_{n}$.

If $(x, y) \in V(G)$ we say $x$ and $y$ are connected in $G$ if there is a path in $G$ from $x$ to $y$. The relation of connection is clearly an equivalence relation. Hence if $V(G)$ is non-null it can be partitioned into disjoint non-null subsets $V_{1}, \cdots, V_{k}$ such that two vertices are connected in $G$ if and only if they belong to the same set $V_{i}$. The subgraphs $G\left[V_{i}\right]$ are the components of $G$. No two of them have an edge or vertex in common and their union is $G$. We write $p_{0}(G)$ for the number of components of $G$. We call $G$ a connected graph if $p_{0}(G)=0$ or 1 . The former case arises only when $E(G)$ and $V(G)$ are both null. Clearly each component of a graph is connected.

If $S \subseteq E(G)$ we define the graphs $G \cdot S, G: S$ and $G \times S$ as in A, $\$ 2$.

Bonds and circuits are defined in $\mathrm{A}, \S 2$. We write $\boldsymbol{C}(G)$ for the class of all subsets $S$ of $E(G)$ such that $G \cdot S$ is a circuit of $G$, and $\boldsymbol{B}(G)$ for the class of all $S \subseteq E(G)$ such that $G \times S$ is a bond of $G$. Thus $S \in C(G)$ if and only if $S$ is the set of edges of some circular path in $G$, and $S \in B(G)$ if and only if there are distinct components $K$ and $L$ of $G:(E(G)-S)$ such that each $A \in S$ has one end (in $G$ ) a vertex of $K$ and the other a vertex of $L$.

(4.1) $B(G)$ and $C(G)$ are regular matroids on $E(G)$.

(4.2) $B(G)=(C(G)) *$.

These theorems can be proved from the results of $A$. In $\mathrm{A}, \S 2$ it was shown that there are two regular chain-groups $\Delta(G)$ and $\Gamma(G)$ on $E(G)$ associated with $G$ (taken with a fixed orientation). By A, (2.4) and (2.7), B(G) and $\boldsymbol{C}(G)$ are the matroids of $\Delta(G)$ and $\Gamma(G)$ respectively. This proves (4.1). By A, (5.5), $\Delta(G)$ and $\Gamma(G)$ are dual chain-groups. Hence (4.2) follows by (2.11).

$$
\begin{aligned}
& d \boldsymbol{B}(G)=\alpha_{0}(G)-p_{0}(G)-1, \\
& d \boldsymbol{C}(G)=\alpha_{1}(G)-\alpha_{0}(G)+p_{0}(G)-1 .
\end{aligned}
$$

To prove (4.3) we observe that by formula (2.2) of A the coboundary of a 0 -chain $f$ on $G$ is zero if and only if in each component of $G$ all the vertices have the same coefficient in $f$. This implies that the maximum number of linearly independent chains of $\Delta(G)$ is $\alpha_{0}(G)-p_{0}(G)$. (4.3) now follows by HII, (2.4). We derive (4.4) from (4.3) by using (4.2) and (2.7), Corollary I. 
If $T$ and $U$ are complementary subsets of $V(G)$ we write $Q(T, U)$ for the set of all edges of $G$ having one end in $T$ and the other in $U$. We note that each $S \in B(G)$ is of the form $Q(T, U)$.

(4.5) If $A \in Q(T, U)$ there exists $S \in B(G)$ such that $A \in S \subseteq Q(T, U)$.

Proof. We can find $S$ such that $A \in S \subseteq Q(T, U), S$ is of the form $Q\left(T^{\prime}, U^{\prime}\right)$, and $\alpha(S)$ has the least value consistent with these conditions. Let $H$ be the component of $G\left[T^{\prime}\right]$ having an end of $A$ in $G$ as a vertex. Then $H$ is the only component of $G\left[T^{\prime}\right]$ having an end of a member of $S$ as a vertex, for otherwise we would have $A \in Q(V(H), V(G)-V(H)) \subset S$, contrary to the definition of $S$. Similarly only one component of $G\left[U^{\prime}\right]$ has an end of a member of $S$ as a vertex. It follows that $S \in B(G)$.

If $S \subseteq E(G)$ we have the following identities.

$$
\begin{aligned}
& C(G \cdot S)=C(G) \times S, \\
& C(G \times S)=C(G) \cdot S, \\
& B(G \cdot S)=B(G) \cdot S, \\
& B(G \times S)=B(G) \times S,
\end{aligned}
$$

Formula (4.6) follows immediately from the definitions. To prove (4.7) suppose $Y \in C(G \times S)$. There is a circular path $P=\left(C_{0}, A_{1}, C_{1}, \cdots, A_{k}, C_{0}\right)$ in $G \times S$ whose edges $A_{i}$ are the members of $Y$. We recall that the vertices of $G \times S$ are components of the graph $G:(E(G)-S)$. For $1 \leqq j \leqq k$ let $x_{j}$ and $y_{j}$ be ends in $G$ of $A_{j}$, distinct if possible, belonging to $C_{j-1}$ and $C_{j}$ respectively $\left(C_{k}=C_{0}\right)$. In $P$ we replace the first term by $x_{1}$, the last by a simple path in the graph $C_{0}$ from $y_{k}$ to $x_{1}$, and each intermediate $C_{i}$ by a simple path in $C_{i}$ from $y_{i}$ to $x_{i+1}$. There results a circular path in $G$. Hence there exists $T \in C(G)$ such that $T \cap S=Y$. Accordingly there exists $Z \in C(G) \cdot S$ such that $Z \subseteq Y$. Conversely suppose $Z \in \mathbf{C}(G) \cdot S$. Then there is a circular path $Q$ $=\left(a_{0}, A_{1}, a_{1}, \cdots, A_{n}, a_{0}\right)$ in $G$ such that those edges $A_{i}$ which belong to $S$ are the members of $Z$. In $Q$ we delete each edge not in $Z$ and its succeeding vertex-term. We then replace each remaining term $a_{i}$ by the component $C_{i}$ of $G:(E(G)-S)$ to which it belongs. There results a re-entrant path $Q^{\prime}$ in $G \times S$ whose edges are the members of $Z$. This must contain a circular path in $G \times S$ as a subsequence. Hence there exists $Y \in C(G \times S)$ such that $Y \subseteq Z$. Formula (4.7) now follows by Axiom I.

To prove (4.8) and (4.9) we take dual matroids in (4.6) and (4.7), apply (2.9) and (2.10), and then use (4.2).

We call a matroid $\boldsymbol{M}$ graphic or cographic if there is a graph $G$ such that $\boldsymbol{M}=\boldsymbol{B}(G)$ or $\boldsymbol{M}=\boldsymbol{C}(G)$ respectively. By the four identities just proved we have:

(4.10) The minors of a graphic (cographic) matroid are graphic (cographic).

A complete 5-graph is a graph having just five vertices $a_{1}, \cdots, a_{5}$ and just ten edges $L_{i j}(1 \leqq i<j \leqq 5)$, the ends of $L_{i j}$ being $a_{i}$ and $a_{j}$. A Thomsen graph is a graph having just six vertices $a_{1}, a_{2}, a_{3}, b_{1}, b_{2}, b_{3}$ and just nine edges 
$L_{i j}(1 \leqq(i, j) \leqq 3)$ such that the ends of $L_{i j}$ are $a_{i}$ and $b_{j}$. Diagrams of these graphs are given in Figure I, the Thomsen graph being shown on the right. We refer to the graphs of these two kinds as the Kuratowski graphs.
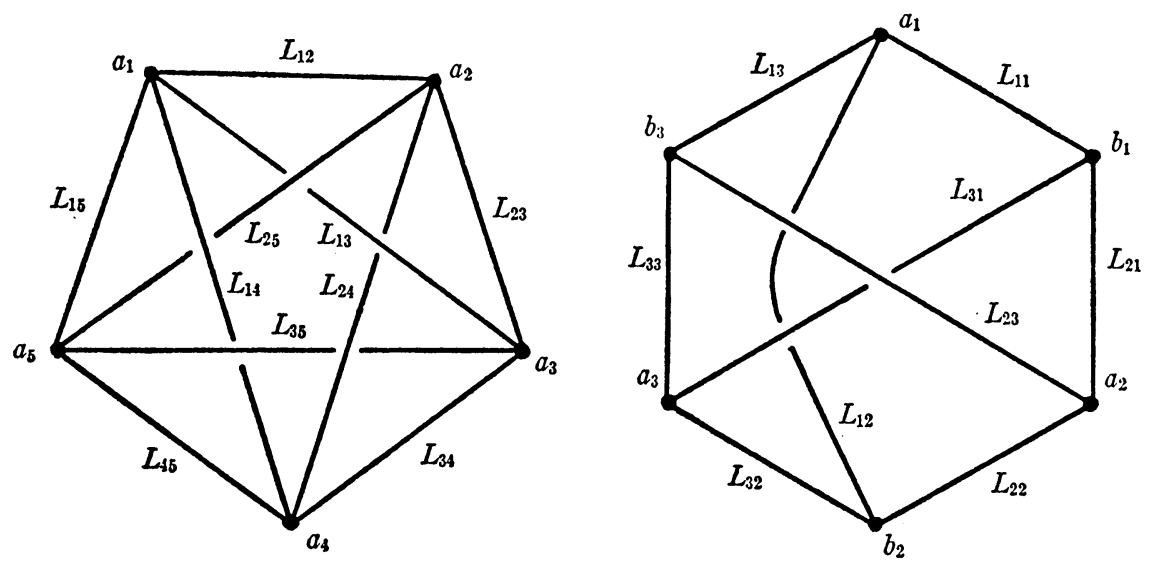

FIG. I

We can now give a more precise statement of the main theorem of the paper. The bond-matroid and circuit-matroid of $G$ mentioned in the Introduction are $\boldsymbol{B}(G)$ and $\boldsymbol{C}(G)$ respectively. The word "contain" used there means "have as a minor."

Theorem. A matroid $\boldsymbol{M}$ is graphic (cographic) if and only if it is regular and has no minor which is the circuit-matroid (bond-matroid) of a Kuratowski graph.

We complete the proof of this theorem in $\S 8$.

Following Hassler Whitney [5] we say $G$ is separable if it is not connected or if there are complementary non-null subsets $S$ and $T$ of $E(G)$ such that $G \cdot S$ and $G \cdot T$ have only one vertex in common.

A subgraph $G \cdot S$ of $G$ is a separate of $G$ if it is non-null, non-separable, and such that no component of $G:(E(G)-S)$ includes more than one vertex of $G \cdot S$. (Separates are called "components" in [5]). By this definition a separate must have at least one edge.

(4.11) Suppose $S \subseteq E(G)$. Then $G \cdot S$ is a separate of $G$ if and only if $S$ is an elementary separator of $B(G)$.

Proof. Suppose $S$ is an elementary separator of $B(G)$. Then $G \cdot S$ is nonnull. Assume $G \cdot S$ separable. Then there are complementary non-null subsets $U$ and $V$ of $S$ such that $G \cdot U$ and $G \cdot V$ have at most one vertex in common. For any $Y \in B(G) \times S$ we have $Y \in B(G) \cdot S=B(G \cdot S)$, by (3.9) and (4.8). Hence there are distinct components $H$ and $K$ of $(G \cdot S):(S-Y)$ such that each $A \in Y$ has one end in $H$ and the other in $K$. Without loss of generality 
we may suppose $H$ includes no common vertex of $G \cdot U$ and $G \cdot V$. It follows that $H$ is a subgraph of one of these and hence that $Y$ is a subset of $U$ or $V$. Thus $U$ is a separator of $B(G) \times S$ and therefore of $B(G)$, contrary to the definition of $S$. We deduce that $G \cdot S$ is nonseparable.

Assume $G:(E(G)-S)$ has a component $L$ which includes two distinct vertices $x$ and $y$ of $G \cdot S$. We can find a simple path $P$ from $x$ to $y$ in $G \cdot S$, and a simple path $Q$ from $y$ to $x$ in $L$. By a proper choice of $y$ and $P$ we can arrange that no vertex in $P$ other than $x$ and $y$ is a vertex of $L$. Combining $P$ and $Q$ to form a circular path in $G$ we see that there exists $X \in \mathbf{C}(G)$ meeting both $S$ and $E(G)-S$. Hence $S$ is not an elementary separator of $\boldsymbol{C}(G)$. This is contrary to the definition of $S$, by (3.10) and (4.2). We conclude that $G \cdot S$ is a separate of $G$.

Conversely suppose $G \cdot S$ is a separate of $G$. Choose $A \in S$ and let $T$ be the elementary separator of $B(G)$ containing $A$. Then $G \cdot T$ is a separate of $G$ by the preceding argument.

Each component of $(G \cdot S):(S-(S \cap T))$ is a subgraph of a component of $G:(E(G)-T)$. It therefore has at most one vertex in common with the subgraph $G \cdot(S \cap T)$ of $G \cdot T$. Since $G \cdot S$ is nonseparable it follows that no component of $(G \cdot S):(S-(S \cap T))$ has an edge, that is $(S \cap T)=S$. A similar argument, with $S$ and $T$ interchanged, shows that $(S \cap T)=T$. Hence $S$ is the elementary separator $T$ of $B(G)$.

If $S \subseteq E(G)$ we call the common vertices of $G \cdot S$ and $G \cdot(E(G)-S)$ the vertices of attachment of $S$, and their number the attachment-number w(S) of $S$ in $G$. In a nonseparable graph no non-null proper subset of $E(G)$ has attachment-number 0 or 1 .

Suppose $S \subseteq E(G)$ and $w(S)=2$. Let the vertices of attachment of $S$ be $x$ and $y$. We construct a graph $G^{\prime}$ such that $E\left(G^{\prime}\right)=E(G), V\left(G^{\prime}\right)=V(G)$ and the same incidence relations hold as in $G$, save only that the incidence with $x$ and $y$ of members of $S$ is governed by the following rule: if $A \in S$ then $A$ is incident with $x(y)$ in $G^{\prime}$ if and only if it is incident with $y(x)$ in $G$. We say that $G^{\prime}$ is obtained from $G$ by reversing $S$. We can recover $G$ from $G^{\prime}$ by reversing $S$ again.

Suppose $Y \in B(G)$. Then $Y=Q(T, U)$ for suitable complementary subsets $T$ and $U$ of $V(G)$. If $x$ and $y$ belong to the same set $T$ or $U$ we write $T^{\prime}=T$ and $U^{\prime}=U$. But if $x$ and $y$ belong to different sets $T$ and $U$ we write $T^{\prime}=(T \cap V(G \cdot(E(G)-S))) \cup(U \cap(V(G \cdot S)-\{x, y\}))$ and $U^{\prime}$ $=(U \cap V(G \cdot(E(G)-S))) \cup(T \cap(V(G \cdot S)-\{x, y\}))$. It is readily verified that in each case $Y=Q\left(U^{\prime}, V^{\prime}\right)$ in $G^{\prime}$. Hence there exists $Y^{\prime} \in B\left(G^{\prime}\right)$ such that $Y^{\prime} \subseteq Y$, by (4.5). Similarly for each $Y^{\prime} \in B\left(G^{\prime}\right)$ there exists $Y \in B(G)$ such that $Y \subseteq Y^{\prime}$. Hence $B\left(G^{\prime}\right)=B(G)$. We thus have:

(4.12) $B(G)$ is invariant under the operation of reversing a subset $S$ of $E(G)$ such that $w(S)=2$.

(4.13) If $Y \in B(G)$ and $B(G) \cdot(E(G)-Y)$ is connected there exists $a \in V(G)$ such that $Y=Q(\{a\}, V(G)-\{a\})$. 
Proof. $G \cdot(E(G)-Y)$ is nonseparable and therefore connected, by (4.8) and (4.11). It is thus a component of $G:(E(G)-Y)$. The other component of this graph including an end of each $A \in Y$ thus has no edge and only one vertex, $a$ say.

5. Convex subclasses of a matroid. The results on convex subclasses of a matroid $M$ given in HI, $\$ 5$ need to be supplemented by the following theorems.

(5.1) Let $\boldsymbol{C}$ and $\boldsymbol{C}^{\prime}$ be convex subclasses of $\boldsymbol{M}$ and let $S$ be a connected flat of $\boldsymbol{M}$ having a point of $\boldsymbol{M}-\boldsymbol{C}$ and a point of $\boldsymbol{M}-\boldsymbol{C}^{\prime}$ as subsets. Then there exists a point of $(\boldsymbol{M}-\boldsymbol{C}) \cap\left(\boldsymbol{M}-\boldsymbol{C}^{\prime}\right)$ on $S$.

Proof. Assume the theorem false. There are points $X$ and $Y$ of $M$ on $S$ such that $X \in \boldsymbol{C} \cap\left(\boldsymbol{M}-\boldsymbol{C}^{\prime}\right)$ and $Y \in \boldsymbol{C}^{\prime} \cap(\boldsymbol{M}-\boldsymbol{C})$. By HI, (5.1), there exists $Z \in M$ on $S$ such that $X \cup Z$ is a connected line of $M$ and $Z \in M-C$. By assumption $Z \in C^{\prime}$. But there is a point of $M$ other than $X$ and $Z$ on $X \cup Z$, by $\mathrm{HI},(3.2)$. By the definition of a convex subclass this point must belong to both $\boldsymbol{M}-\boldsymbol{C}$ and $\boldsymbol{M}-\boldsymbol{C}^{\prime}$.

(5.2) If $\boldsymbol{M}$ is binary and $S$ is any set of cells of $\boldsymbol{M}$ then the class $\boldsymbol{C}$ of all $X \in M$ such that $S \subseteq X$ or $X \cap S=\varnothing$ is a convex subclass of $\boldsymbol{M}$.

Proof. Let $X, Y$ and $Z$ be the three points on some connected line $L$ of $\boldsymbol{M}$. (HII, (2.7)). Suppose $(X, Y) \in C$. Then $Z \in C$, for $Z$ is the $\bmod 2$ sum of $X$ and $Y$ by HII, (2.6) and $X \cap Y \neq \phi$ by HI, (3.1).

6. Properties of a matroid at a point. In this section and the next we study the relation of a point $Y$ of a matroid $M$ on a set $M$ to the rest of the matroid.

$$
d(\boldsymbol{M} \cdot(M-Y))=d \boldsymbol{M}-1 .
$$

Proof. Choose $a \in Y$ and let $D$ be a dendroid of $M \cdot(M-Y)$. Then $D \cup\{a\}$ meets each $X \in \boldsymbol{M}$. For each $b \in D$ there exists $Z_{b} \in \boldsymbol{M} \cdot(M-Y)$ such that $Z_{b} \cap D=\{b\}$. Hence there exists also $X_{b} \in M$ such that $X_{b} \cap D=\{b\}$. By Axiom II we can arrange that $a \notin X_{b}$. Hence no proper subset of $D \cup\{a\}$ meets each $X \in M$ and so $D \cup\{a\}$ is a dendroid of $\boldsymbol{M}$. The theorem follows by (2.2).

(6.2) Suppose $S \subseteq M-Y$ and $d$ is an integer $\geqq-1$. Then $S$ is a d-flat of $\boldsymbol{M} \cdot(M-Y)$ if and only if $S \cup Y$ is a $(d+1)$-flat of $\boldsymbol{M}$.

Proof. It is clear that if $S$ is a flat of $\boldsymbol{M} \cdot(M-Y)$ then $S \cup Y$ is a flat of $\boldsymbol{M}$. The converse is also true, by HII, (3.1). To complete the proof we observe that $d((\boldsymbol{M} \cdot(M-Y)) \times S)=d((\boldsymbol{M} \times(S \cup Y)) \cdot S)=d(M \times(S \cup Y))-1$, by (3.3) and (6.1).

(6.3) Let $S$ be a connected flat of $\boldsymbol{M} \cdot(M-Y)$. Then either $S \cup Y$ is a connected flat of $\boldsymbol{M}$ or $(\boldsymbol{M} \cdot(M-Y)) \times S=\boldsymbol{M} \times S$.

Proof. Since $S$ is connected in $\boldsymbol{M} \cdot(M-Y)$ the only possible separation of the flat $S \cup Y$ of $M$ is $\{S, Y\}$ (HI, $\S 4$ ). If such a separation exists we have $(\boldsymbol{M} \cdot(M-Y)) \times S=(\boldsymbol{M} \times(S \cup Y)) \cdot S=(\boldsymbol{M} \times(S \cup Y)) \times S=\boldsymbol{M} \times S, \quad$ by (3.1), (3.3) and (3.9). 
From now on we suppose $M$ binary. If $Z \in M \cdot(M-Y)$ then $Y \cup Z$ is a line of $M$, by (6.2). If it is connected it has just two points other than $Z$, by HII, (2.7). Their intersections with $Y$ are complementary non-null subsets $T$ and $U$ of $Y$, by HII, (2.6). We call the unordered pair $\{T, U\}$ the partition of $Y$ determined by $Z$. If $Y \cup Z$ is not connected its two points (HI, (3.2)) must be $Y$ and $Z$. In this case we define the partition of $Y$ determined by $Z$ as the unordered pair $\{Y, \varnothing\}$.

We say that $Z$ cuts a subset $S$ of $Y$ if it determines a partition $\{T, U\}$ of $Y$ such that $\varnothing \subset S \cap T \subset S$.

(6.4) If $\boldsymbol{M}$ is binary the points of $\boldsymbol{M} \cdot(M-Y)$ which do not cut a given subset $S$ of $Y$ constitute a convex subclass of $\boldsymbol{M} \cdot(M-Y)$.

Proof. Let $Y_{1}, Y_{2}$ and $Y_{3}$ be the three points on a connected line $L$ of $\boldsymbol{M} \cdot(M-Y)$. Suppose $Y_{1}$ and $Y_{2}$ do not cut $S$. Then each point of $\boldsymbol{M}$ on the lines $Y \cup Y_{1}$ and $Y \cup Y_{2}$ of $M$ either contains $S$ or does not meet $S$. If $X_{3}$ is a point on the line $Y \cup Y_{3}$ of $\boldsymbol{M}$ other than $Y$ there is a line $L^{\prime}$ of $\boldsymbol{M}$ on $X_{3}$ and the plane $L \cup Y$ which is not on $Y$, by HI, (2.4). This meets $Y \cup Y_{1}$ and $Y \cup Y_{2}$ in distinct points, by $\mathrm{HI},(2.2)$ and (2.5). Hence $X_{3}$ either contains $S$ or does not meet $S$, by (5.2). It follows that $Y_{3}$ does not cut $S$.

We refer to the elementary separators of $\boldsymbol{M} \cdot(M-Y)$ as the bridges of $Y$ in $\boldsymbol{M}$. A bridge of $Y$ having only one cell is unicellular. If $\boldsymbol{M}=\boldsymbol{B}(G)$ the bridges of $Y$ correspond to the separates of $G \cdot(E(G)-Y)$, by (4.8) and (4.11). If $\boldsymbol{M}=\boldsymbol{C}(G)$ they correspond to the separates of $G \times(E(G)-Y)$, by (3.10), (4.2), (4.7) and (4.11). In the Thomsen graph of Figure I for example $\left\{L_{12}\right\}$ is a unicellular bridge of the point $\left\{L_{11}, L_{21}, L_{22}, L_{32}, L_{33}, L_{13}\right\}$ of $\boldsymbol{C}(G)$. It determines the partition $\left\{\left\{L_{11}, L_{21}, L_{22}\right\},\left\{L_{32}, L_{33}, L_{13}\right\}\right\}$.

Two points $Z$ and $Z^{\prime}$ of $\boldsymbol{M} \cdot(M-Y)$ are skew with respect to $Y$ if they determine partitions $\{T, U\}$ and $\left\{T^{\prime}, U^{\prime}\right\}$ of $Y$ such that the intersections $T \cap T^{\prime}, T \cap U^{\prime}, U \cap T^{\prime}$ and $U \cap U^{\prime}$ are all non-null. Two bridges $B$ and $B^{\prime}$ of $Y$ in $\boldsymbol{M}$ are skew if there are points $Z$ and $Z^{\prime}$ of $\boldsymbol{M} \cdot(M-Y)$, skew with respect to $Y$, such that $Z \subseteq B$ and $Z^{\prime} \subseteq B^{\prime}$. We also say that a point $Z$ of $\boldsymbol{M} \cdot(M-Y)$ is skew to a bridge $B$ of $Y$ in $\boldsymbol{M}$ if there is a point $Z^{\prime}$ of $\boldsymbol{M} \cdot(M-Y)$ on $B$ which is skew to $Z$.

(6.5) Let $B_{1}, B_{2}$ and $B_{3}$ be distinct bridges of $Y$ in $M$ such that $B_{2}$ is skew to both $B_{1}$ and $B_{3}$. Then either there is a point $Z_{2}$ of $\boldsymbol{M} \cdot(M-Y)$ on $B_{2}$ which is skew to both $B_{1}$ and $B_{3}$ or there are points $Z_{1}$ and $Z_{3}$ of $\boldsymbol{M} \cdot(M-Y)$ on $B_{1}$ and $B_{3}$ respectively such that $Z_{1}, B_{2}$ and $Z_{3}$ are mutually skew.

Proof. There are points $Z_{1}, Z_{2}^{\prime}, Z_{2}^{\prime \prime}$ and $Z_{3}$ of $\boldsymbol{M} \cdot(M-Y)$ on $B_{1}, B_{2}, B_{2}$ and $B_{3}$ respectively such that $Z_{1}$ is skew to $Z_{2}^{\prime}$ and $Z_{2}^{\prime \prime}$ is skew to $Z_{3}$. If $Z_{1}$ and $Z_{3}$ are skew the second alternative of the theorem holds. In the remaining case $Z_{1}$ and $Z_{3}$ determine partitions $\left\{S_{1}, T_{1}\right\}$ and $\left\{S_{3}, T_{3}\right\}$ of $Y$ such that $T_{1} \cap T_{3}=\varnothing$, i.e., $T_{1} \subseteq S_{3}$ and $T_{3} \subseteq S_{1}$.

Now $Z_{2}^{\prime}$ cuts $T_{1}, Z_{2}^{\prime \prime}$ cuts $T_{3}$ and $B_{2}$ is a connected flat of $M \cdot(M-Y)$, Hence, by (5.1) and (6.4) there exists $Z_{2} \in M \cdot(M-Y)$ on $B_{2}$ cutting both $T_{1}$ and $T_{3}$. Then $Z_{2}$ is skew to both $Z_{1}$ and $Z_{3}$, and therefore to both $B_{1}$ and $B_{3}$. 
7. Regular matroids. In HII, §4, we defined geometrical figures of Types $\mathrm{BI}$ and BII. In the main theorem of HII we showed that a binary matroid is regular if and only if it has no figure of Type BI or BII. We make use of this theorem in the proofs of (7.1) and (7.2).

We suppose given a regular matroid $\boldsymbol{M}$ on a set $M$, and some $Y \in M$.

(7.1) Let $Z_{1}$ and $Z_{2}$ be distinct points on a connected line $L$ of $\boldsymbol{M} \cdot(M-Y)$. Then $Z_{1}$ and $Z_{2}$ are not skew with respect to $Y$.

Proof. $L \cup Y$ is a plane $P$ of $M$, by (6.2). It includes three distinct lines $Y \cup Z_{1}, Y \cup Z_{2}$ and $Y \cup Z_{3}$ of $M$ on $Y$, where $Z_{3}$ is the third point of $M \cdot(M-Y)$ on $L$.

Assume $Z_{1}$ and $Z_{2}$ skew. They determine partitions $\left\{S_{1}, T_{1}\right\}$ and $\left\{S_{2}, T_{2}\right\}$ of $Y$ respectively. There are cells $a \in S_{1} \cap S_{2}, b \in S_{1} \cap T_{2}, c \in T_{1} \cap S_{2}$ and $d \in T_{1} \cap T_{2}$. The flats $\langle P-\{a\}\rangle,\langle P-\{b\}\rangle,\langle P-\{c\}\rangle$ and $\langle P-\{d\}\rangle$ of $\boldsymbol{M}$ are lines on $P$ which are not on $Y$, by HI, (2.3). It is easily seen that they are distinct. For example $\langle P-\{a\}\rangle$ is the only one which is on the points $Z_{1} \cup T_{1}$ and $Z_{2} \cup T_{2}$ of $M$.

As there are seven distinct lines on $P$ the matroid $M$ has a figure of Type BI. This is impossible since $M$ is regular.

(7.2) Let $Z_{1}, Z_{2}$ and $Z_{2}$ be points of $\boldsymbol{M} \cdot(M-Y)$ on distinct bridges $B_{1}, B_{2}$ and $B_{3}$ respectively of $Y$ in $M$. Let $Z_{i}$ determine the partition $\left\{S_{i}, T_{i}\right\}$ of $Y(i=1,2,3)$. Then if $S_{3}$ meets both $S_{1} \cap S_{2}$ and $T_{1} \cap T_{2}$ it contains one of the sets $S_{1} \cap T_{2}$ and $S_{2} \cap T_{1}$.

Proof. If the theorem is false we can choose $K=\{a, b, c, d, e, f, g\} \subseteq M$ such that $a \in Z_{1}, b \in Z_{2}, c \in Z_{3}, d \in S_{1} \cap S_{2} \cap S_{3}, e \in T_{1} \cap T_{2} \cap S_{3}, f \in S_{1} \cap T_{2}$, $g \in S_{2} \cap T_{1}, f \notin S_{3}, g \notin S_{3}$.

In the matroid $\boldsymbol{M} \cdot(M-Y)$ the flats $Z_{1} \cup Z_{2}, Z_{2} \cup Z_{3}$ and $Z_{3} \cup Z_{1}$ are lines, and $Z_{1} \cup Z_{2} \cup Z_{3}$ is a plane. This follows from the definition of dimension in HI, §1. Hence $Y \cup Z_{1} \cup Z_{2} \cup Z_{3}$ is a 3 -flat $E$ of $M$, by (6.2). There are points of $M$ on $E$ whose intersections with $K$ are $\{a, d, f\},\{a, g, e\},\{b, d, g\}$, $\{b, e, f\},\{c, f, g\},\{c, d, e\}$ and $\{a, b, c\}$. For example $\left(Z_{1} \cup S_{1}\right) \cap K=\{a, d, f\}$, and similar verifications may be made for the next five intersections. As for $\{a, b, c\}$ we observe that $Z_{1} \cup T_{1} \subset Z_{1} \cup T_{1} \cup Z_{2} \cup T_{2} \subset Z_{1} \cup T_{1} \cup Z_{2} \cup T_{2} \cup Z_{3}$ $\cup T_{3} \subset E$. Hence $Z_{1} \cup T_{1} \cup Z_{2} \cup T_{2}$ is a line of $M$, by HI, (2.2). Its points are $Z_{1} \cup T_{1}, Z_{2} \cup T_{2}$ and their $\bmod 2$ sum, $Q$ say. $Q \cap K=\{a, b, f, g\}$. Further $Z_{1} \cup T_{1} \cup Z_{2} \cup T_{2} \cup Z_{3} \cup T_{3}$ is a plane $P$ of $M$ on $E$. The line $\langle P-\{e\}\rangle$ of $\boldsymbol{M}$ is on the points $Z_{3} \cup T_{3}$ and $Q$. As these distinct points have a common cell $f$ their $\bmod 2$ sum, $R$ say, is a third point of $M$ on $\langle P-\{e\}\rangle$. But $R \cap K=$ $\{a, b, c\}$.

Considering the seven intersections with $K$ set out above, we see that each cell of $K$ occurs in just three of them, and that no two have two cells in common. Hence given any three cells $x, y$ and $z$ of $K$ we can find a point of $M$ on $E$ including $x$ but not $y$ or $z$. It follows that no three of the planes $\langle E-\{x\}\rangle,(x \in K)$, of $\boldsymbol{M}$ have a common line; we can find a point on any 
two of them which is not on the third. Hence these seven planes on $E$ are distinct and define a figure of Type BII. This is impossible since $M$ is regular.

If $B$ is any bridge of $Y$ in $\boldsymbol{M}$ we define $\pi(\boldsymbol{M}, B, Y)$ as the class of all minimal non-null subsets of $Y$ which are intersections of points of $M \times(B \cup Y)$.

(7.3) The members of $\boldsymbol{\pi}(\boldsymbol{M}, B, Y)$ are disjoint and their union is $Y$. Moreover if $W \in \pi(\boldsymbol{M}, B, Y)$ then either $W=Y$ or there exists $Z \in \boldsymbol{M} \cdot(M-Y)$ on $B$ such that $W \cup Z \in M \times(B \cup Y)$.

Proof. Let $a$ be any cell of $Y$. If $(\boldsymbol{M} \cdot(M-Y)) \times B$ is non-null let $U$ be a subset of $Y$ such that $a \in U$, some $Z \in M \cdot(M-Y)$ on $B$ determines the partition $\{U, Y-U\}$ of $Y$, and $\alpha(U)$ has the least value consistent with these conditions. If $(\boldsymbol{M} \cdot(M-Y)) \times B$ is null we write $U=Y$.

Assume some $W \in \pi(M, B, Y)$ satisfies $\varnothing \subset W \cap U \subset U$. Then there exists $X \in M \times(B \cup Y)$ such that $\varnothing \subset X \cap U \subset U$. Clearly $X \cup Y$ is a connected flat of $M \times(B \cup Y)$. By (5.2) and HI, (5.1), we can find $X^{\prime} \in M \times(B \cup Y)$ on $X \cup Y$ such that $\varnothing \subset X^{\prime} \cap U \subset U$ and $X^{\prime} \cup Y$ is a connected line of $M \times(B \cup Y)$. Then, by (6.2), $X^{\prime}-\left(X^{\prime} \cap Y\right)$ is a point of $M \cdot(M-Y)$ on $B$. It determines the partition $\left\{X^{\prime} \cap Y, Y-\left(X^{\prime} \cap Y\right)\right\}$ of $Y$, and so cuts $U$. We note that $(M \cdot(M-Y))$ $X B$ is non-null and so $Z$ is defined. By (6.4) and HI, (5.1), we can find $Z^{\prime} \in \boldsymbol{M} \cdot(M-Y)$ on $B$ which cuts $U$ and is such that $Z \cup Z^{\prime}$ is a connected line of $\boldsymbol{M} \cdot(M-Y)$. By (7.1) $Z^{\prime}$ determines a partition $\{S, Y-S\}$ of $Y$ such that $S \subset U$. We have $a \in U-S$, by the definition of $U$. Choose $b \in S$.

By (6.2) $Y \cup Z \cup Z^{\prime}$ is a plane $P$ of $M$. The line $\langle P-\{b\}\rangle$ of $M$ is on the points $Z \cup(Y-U)$ and $Z^{\prime} \cup(Y-S)$ of $M$. Since these are distinct and not disjoint their $\bmod 2$ sum, $Q$ say, is also a point of $M$ on $\langle P-\{b\}\rangle$. But the $\bmod 2$ sum of $Z$ and $Z^{\prime}$ is the third point of $M \cdot(M-Y)$ on the line $Z \cup Z^{\prime}$. It determines the partition $\{Q \cap Y, Y-(Q \cap Y)\}=\{U-S, Y-(U-S)\}$ of $Y$. This contradicts the definition of $U$.

We deduce from this contradiction that $U$ is itself a member of $\boldsymbol{\pi}(\boldsymbol{M}, B, Y)$ and that it meets no other member. Since $a$ may be any cell of $Y$ the theorem follows.

While discussing the next three theorems we bear in mind that the minors of $M$ are regular matroids (3.7).

(7.4) Let $B$ be a bridge of $Y$ in $M$ and $S$ a subset of $M$ such that $B \cup Y \subseteq S$. Then $B$ is a bridge of $Y$ in $\boldsymbol{M} \times S$, and $\boldsymbol{\pi}(\boldsymbol{M} \times S, B, Y)=\boldsymbol{\pi}(\boldsymbol{M}, B, Y)$.

Proof. $(M \times S) \cdot(S-Y)=(\boldsymbol{M} \cdot(M-Y)) \times(S-Y)$, by (3.4). Hence $B$ is a separator of $(\boldsymbol{M} \times S) \cdot(S-Y)$, by (3.8). Moreover $((\boldsymbol{M} \times S) \cdot(S-Y)) \times B$ $=((\boldsymbol{M} \cdot(M-Y)) \times(S-Y)) \times B=(\boldsymbol{M} \cdot(M-Y)) \times B$, by (3.1), and this matroid is connected. Hence $B$ is a bridge of $Y$ in $\boldsymbol{M} \times S$. Since $(\boldsymbol{M} \times S) \times(B \cup Y)$ $=\boldsymbol{M} \times(B \cup Y)$, by (3.1), the theorem follows.

(7.5) Let $B$ be a bridge of $Y$ in $M$. Let $S$ be a subset of $M$ such that $B \cup Y \subseteq S$ and no $Z \in M \cdot(M-Y)$ is a subset of $M-S$. Then $Y \in M \cdot S, B$ is a bridge of $Y$ in $\boldsymbol{M} \cdot S$, and $\boldsymbol{\pi}(\boldsymbol{M} \cdot S, B, Y)=\boldsymbol{\pi}(\boldsymbol{M}, B, Y)$.

Proof. $(\boldsymbol{M} \cdot S) \cdot(S-Y)=(\boldsymbol{M} \cdot(M-Y)) \cdot(S-Y)$ by (3.2). Hence $B$ is a 
separator of $(\boldsymbol{M} \cdot S) \cdot(S-Y)$, by (3.8). Moreover $((\boldsymbol{M} \cdot S) \cdot(S-Y)) \cdot B$ $=(\boldsymbol{M} \cdot(M-Y)) \cdot B$, by $(3.2)$, and this matroid is connected. Hence $B$ is a bridge of $Y$ in $\boldsymbol{M} \cdot S$ if $Y \in \boldsymbol{M} \cdot S$.

Suppose some $X \in M \times((M-S) \cup B \cup Y)$ meets $M-S$. Then, by HI, (3.1), some $Z \in M \cdot(M-Y)$ meets $M-S$ and satisfies $Z \subseteq(M-S) \cup B$. But then $Z \subseteq M-S$, since $B$ is a separator of $\boldsymbol{M} \cdot(M-Y)$, and this is contrary to the definition of $S$. We thus have $(\boldsymbol{M} \cdot S) \times(B \cup Y)=(\boldsymbol{M} \times((M-S) \cup B \cup Y))$ $\cdot(B \cup Y)$, by $(3.3),=(\boldsymbol{M} \times(B \cup Y)) \cdot(B \cup Y)=\boldsymbol{M} \times(B \cup Y)$. We note that this implies $Y \in M \cdot S$. The theorem follows.

(7.6) Let $B$ be any bridge of $Y$ in $M$. Let $S$ be a subset of $M$ such that $M-Y \subset S$. Then $S \cap Y \in M \cdot S, B$ is a bridge of $S \cap Y$ in $M \cdot S$, and

$$
\boldsymbol{\pi}(\boldsymbol{M} \cdot S, B, S \cap Y)
$$

is the class of all non-null intersections with $S$ of members of $\boldsymbol{\pi}(\boldsymbol{M}, B, Y)$.

Proof. There exists $Y^{\prime} \in M \cdot S$ such that $Y^{\prime} \subseteq S \cap Y$ and $Y^{\prime \prime} \in M$ such that $Y^{\prime \prime} \cap S=Y^{\prime}$. But then $Y^{\prime \prime} \subseteq Y$. Hence $Y^{\prime \prime}=Y$ and $S \cap Y=Y^{\prime} \in \boldsymbol{M} \cdot S$ by Axiom I.

$(\boldsymbol{M} \cdot S) \cdot(S-(S \cap Y))=\boldsymbol{M} \cdot(S-(S \cap Y))=\boldsymbol{M} \cdot(M-Y)$, by (3.2). Hence $B$ is a bridge of $S \cap Y$ in $M \cdot S$.

Suppose $W \in \boldsymbol{\pi}(M, B, Y)$ and $W \cap S \neq \varnothing$. By (7.3) there exists $X \in \boldsymbol{M}$ $\times(B \cup Y)$ such that $X \cap Y=W$. By HII, (3.1), there exists $X^{\prime} \in(\boldsymbol{M} \cdot S)$ $\times(B \cup(S \cap Y))$ such that $\varnothing \subset X^{\prime} \cap Y \subseteq X \cap S \cap Y=W \cap S$. Hence there exists $W^{\prime} \in \boldsymbol{\pi}(\boldsymbol{M} \cdot S, B, S \cap Y)$ such that $W^{\prime} \subseteq W \cap S$.

Conversely, suppose $W^{\prime} \in \boldsymbol{\pi}(\boldsymbol{M} \cdot S, B, S \cap Y)$. By (7.3) there exists $X^{\prime} \in(M \cdot S) \times(B \cup(S \cap Y))$ such that $X^{\prime} \cap(S \cap Y)=W^{\prime}$. Hence there exists $X \in M \times(B \cup Y)$ such that $X \cap(S \cap Y)=W^{\prime}$. Hence, by (7.3), there exists $W \in \pi(\boldsymbol{M}, B, Y)$ such that $\varnothing \subset W \cap S \subseteq W^{\prime}$.

The foregoing results, together with (7.3), imply (7.6).

(7.7) Let $B_{1}$ and $B_{2}$ be distinct bridges of $Y$ in $\boldsymbol{M}$. Let $W_{1} \in \boldsymbol{\pi}\left(\boldsymbol{M}, B_{1}, Y\right)$ and $W_{2} \in \boldsymbol{\pi}\left(\boldsymbol{M}, B_{2}, Y\right)$ be such that $W_{2} \subseteq W_{1}$. Let $Z \in \boldsymbol{M} \cdot(M-Y)$ on $B_{2}$ be such that $Z \cup W_{2} \in M$, so that $Z \cup\left(Y-W_{2}\right),=Y^{\prime}$ say, is a point of $\boldsymbol{M}$. Then $B_{1}$ is a bridge of $Y^{\prime}$ in $M$.

Proof. $\left(\boldsymbol{M} \cdot\left(M-Y^{\prime}\right)\right) \cdot B_{1}=(\boldsymbol{M} \cdot(M-Y)) \cdot B_{1}$, by (3.2). As this matroid is connected $B_{1}$ is a subset of a bridge $B^{\prime}$ of $Y^{\prime}$ in $M$, by (3.8).

Suppose $Z_{1} \in M \cdot\left(M-Y^{\prime}\right)$ on $B^{\prime}$ and $Z_{1} \cap B_{1} \neq \varnothing$. There exists $X \in M$ $\times\left(B^{\prime} \cup Y^{\prime}\right)$ such that $X \cap B^{\prime}=Z_{1}$. By HII, (3.1), there exists $Z_{2} \in M \cdot(M-Y)$ such that $Z_{2} \cap B_{1} \neq \varnothing$ and $Z_{2} \subseteq X \cap(M-Y)$. This implies $Z_{2} \cap B_{1} \subseteq Z_{1}$. But $B_{1}$ is a separator of $M \cdot(M-Y)$, and so $Z_{2} \subseteq Z_{1} \cap B_{1}$. Since $Z_{2}$ is on $B_{1}$ it determines a partition $\{S, T\}$ of $Y$ such that $W_{2} \subseteq W_{1} \subseteq T$. Since $Z_{2} \cup S \in M$ there exists $Z_{3} \in M \cdot\left(M-Y^{\prime}\right)$ such that $Z_{3} \subseteq\left(Z_{2} \cup S\right) \cap\left(M-Y^{\prime}\right) \subseteq Z_{2} \subseteq Z_{1} \cap B_{1}$. Then $Z_{1}=Z_{3}$, by Axiom $\mathrm{I}$, and so $Z_{1} \subseteq B_{1}$. We deduce that $B_{1}$ is a non-null separator of $\left(\boldsymbol{M} \cdot\left(M-Y^{\prime}\right)\right) \times B^{\prime}$, and therefore $B_{1}=B^{\prime}$. 
We write $\beta(\boldsymbol{M}, Y)$ for the number of bridges of $Y$ in $\boldsymbol{M}$. An $n$-bridge of $Y$ in $\boldsymbol{M}$ is a bridge $B$ of $Y$ in $\boldsymbol{M}$ such that $\alpha(\boldsymbol{\pi}(\boldsymbol{M}, B, Y))=n$. Two $n$-bridges $B_{1}$ and $B_{2}$ of $Y$ in $M$ are equivalent if $\boldsymbol{\pi}\left(\boldsymbol{M}, B_{1}, Y\right)=\pi\left(\boldsymbol{M}, B_{2}, Y\right)$. Two bridges $B_{1}$ and $B_{2}$ of $Y$ in $M$ overlap if the equation $W_{1} \cup W_{2}=Y$ is false whenever $W_{1} \in \boldsymbol{\pi}\left(\boldsymbol{M}, B_{1}, Y\right)$ and $W_{2} \in \boldsymbol{\pi}\left(\boldsymbol{M}, B_{2}, Y\right)$. For example any two skew bridges overlap.

(7.8) If $\boldsymbol{M}$ is any regular matroid such that $d \boldsymbol{M} \geqq 2$ then there exists $Y \in M$ such that $\beta(M, Y) \geqq 2$.

Proof. Assume the theorem false. Choose $Y \in M$. Then $\beta(M, Y)=1$ since $d \boldsymbol{M}>0$. Thus $M-Y$ is a bridge of $Y$ in $\boldsymbol{M}$.

Choose $W \in \boldsymbol{\pi}(\boldsymbol{M}, M-Y, Y)$. There exists $Z \in \boldsymbol{M} \cdot(M-Y)$ determining the partition $\{W, Y-W\}$ of $Y$, by (6.1) and (7.3). Then $Y \cup Z$ is a line of $\boldsymbol{M}$, possibly disconnected, and $Z \cup(Y-W),=Y^{\prime}$ say, is a point of $\boldsymbol{M}$. We have $M-(Y \cup Z) \neq \varnothing$ since $d M>1$.

By (6.2) $W$ is a point of $\boldsymbol{M} \cdot\left(M-Y^{\prime}\right)$. Suppose $Z^{\prime} \in \boldsymbol{M} \cdot\left(M-Y^{\prime}\right)$ and $Z^{\prime} \cap W \neq \varnothing$. Then there exists $X \in M$ such that $Z^{\prime}=X \cap\left(M-Y^{\prime}\right)$ and so $X \cap W=Z^{\prime} \cap W$. Hence $W \subseteq Z^{\prime}$ since $W \in \pi(\boldsymbol{M}, M-Y, Y)$, and so $Z^{\prime}=W$ by Axiom I. Hence $W$ is a separator of $\boldsymbol{M} \cdot\left(M-Y^{\prime}\right)$. But $\left(M-Y^{\prime}\right)-W$ $=M-(Y \cup Z) \neq \varnothing$. Hence $\beta\left(\boldsymbol{M}, Y^{\prime}\right) \geqq 2$, contrary to assumption.

(7.9) Let $B_{1}$ and $B_{2}$ be overlapping bridges of a point $Y$ of the regular matroid $M$. Then either $B_{1}$ and $B_{2}$ are skew with respect to $Y$ or they are equivalent 3-bridges.

Proof. Suppose $B_{1}$ and $B_{2}$ are not equivalent $n$-bridges. Without loss of generality we may suppose $\varnothing \subset W_{1} \cap W_{2} \subset W_{2}$ for some $W_{1} \in \boldsymbol{\pi}\left(\boldsymbol{M}, B_{1}, Y\right)$ and $W_{2} \in \pi\left(\boldsymbol{M}, B_{2}, Y\right)$. We can find $U \in \boldsymbol{\pi}\left(\boldsymbol{M}, B_{1}, Y\right)$ such that $\varnothing \subset U$ $\cap\left(Y-W_{2}\right) \subset Y-W_{2}$. For otherwise some $U^{\prime} \in \pi\left(\boldsymbol{M}, B_{1}, Y\right)$ satisfies $Y-W_{2}$ $\subseteq U^{\prime}$, whence $U^{\prime} \cup W_{2}=Y$, contrary to hypothesis. Thus, by (7.3), there are points $Z_{1}$ and $Z_{2}$ of $\boldsymbol{M} \cdot(M-Y)$ on $B_{1}$ cutting $W_{2}$ and $Y-W_{2}$ respectively. By (5.1) and (6.4) some point of $\boldsymbol{M} \cdot(M-Y)$ on $B_{1}$ cuts both these sets. Hence $B_{1}$ and $B_{2}$ are skew, by (7.3).

Suppose next that $B_{1}$ and $B_{2}$ are equivalent $n$-bridges. Then $n \geqq 3$ since the bridges overlap. If $n>3$ we choose distinct members $W_{1}, W_{2}, W_{3}$ and $W_{4}$ of $\boldsymbol{\pi}\left(\boldsymbol{M}, B_{1}, Y\right)=\boldsymbol{\pi}\left(\boldsymbol{M}, B_{2}, Y\right)$. Applying (5.1), (6.4) and (7.3) as before we find there exists $Z \in \boldsymbol{M} \cdot(M-Y)$ on $B_{1}$ cutting both $W_{1} \cup W_{2}$ and $W_{3} \cup W_{4}$. We may suppose without loss of generality that $Z$ determines a partition $\{S, T\}$ of $Y$ such that $W_{1} \cup W_{3} \subseteq S$ and $W_{2} \cup W_{4} \subseteq T$. A similar argument shows that some $Z^{\prime} \in M \cdot(M-Y)$ on $B_{2}$ cuts both $W_{1} \cup W_{3}$ and $W_{2} \cup W_{4}$. Hence $B_{1}$ and $B_{2}$ are skew.

8. Proof of the main theorem. Let $G$ be any graph and $Y$ any point of $\boldsymbol{B}(G)$. Of the components of $G:(E(G)-Y)$ just two include ends in $G$ of members of $Y$. We call these two the end-graphs of $Y$ in $G$.

Let $B$ be any bridge of $Y$ in $B(G)$. It is an elementary separator of $\boldsymbol{B}(G) \cdot(E(G)-Y)$, that is $\boldsymbol{B}(G \cdot(E(G)-Y))$ by (4.8). Hence $G \cdot B$ is a separate 
of $G \cdot(E(G)-Y)$, by (4.11). It follows that $G \cdot B$ is a separate of some component $H$ of $G:(E(G)-Y)$.

If $v \in V(G \cdot B)$ we write $C(B, v)$ for the component of $H:(E(H)-B)$ having $v$ as a vertex. We also write $Y(B, v)$ for the set of all $A \in Y$ such that one end of $A$ in $G$ is a vertex of $C(B, v)$.

We recall that $B(G)$ is regular, by (4.1).

(8.1) If $G \cdot B$ is a separate of an end-graph $H$ of $Y$ in $G$ then $\pi(B(G), B, Y)$ is the class of all $Y(B, v)$ such that $v \in V(G \cdot B)$ and $Y(B, v) \neq \varnothing$. In the remaining case $\pi(B(G), B, Y)=\{Y\}$.

Proof. Let $H$ be the component of $G:(E(G)-Y)$ having $G \cdot B$ as a separate. Suppose $X \in B(G) \times(B \cup Y)$. We can write $X=Q(T, U)$ for suitable complementary subsets $T$ and $U$ of $V(G)$. If $K$ is a component other than $H$ of $G:(E(G)-Y)$ then $v(K)$ is a subset of $T$ or $U$ since $E(K) \cap X=\varnothing$. Similarly $V(C(B, v))$ is a subset of $T$ or $U$ for each $v \in V(G \cdot B)$. If $H$ is not an end-graph of $Y$ it follows that $X \cap Y=\varnothing$ or $Y$. We then deduce that $\boldsymbol{\pi}(\boldsymbol{B}(G), B, Y)=\{Y\}$. If $H$ is an end-graph of $Y$ we may suppose the vertices of the other end-graph to belong to $U$. Then $X \cap Y$ is the union of the sets $Y(B, v)$ such that $v \in T \cap V(G \cdot B)$. Moreover if $v \in V(G \cdot B)$ and $Y(B, v) \neq \varnothing$ we can find $A \in Y(B, v)$ and $X \in B(G) \times(B \cup Y)$ such that

$$
A \in X \subseteq Q(V(C(B, v)), V(G)-V(C(B, v))),
$$

by (4.5). Then $A \in X \cap Y \subseteq Y(B, v)$. It follows that $\boldsymbol{\pi}(\boldsymbol{B}(G), B, Y)$ is the class of all non-null $Y(B, v)$, as required.

Let $\boldsymbol{M}$ be any regular matroid. We say that a point $Y$ of $\boldsymbol{M}$ is even if the bridges of $Y$ in $M$ can be arranged in two disjoint classes so that no two members of the same class overlap. If this arrangement is impossible $Y$ is odd. Clearly $Y$ is odd if and only if there is a cyclic sequence $P=\left(B_{1}, B_{2}, \cdots\right.$, $\left.B_{2 n+1}, B_{1}\right)$ of an odd number $2 n+1$, where $n \geqq 1$, of distinct bridges of $Y$ in $\boldsymbol{M}$ such that each $B_{i}$ overlaps its two neighbours in $P$. We refer to such a sequence as an odd overlap-circuit of $Y$.

(8.2) In a graphic matroid every point is even.

Proof. Let $Y$ be any point of a graphic matroid $\boldsymbol{M}$. There is a graph $G$ such that $\boldsymbol{M}=\boldsymbol{B}(G)$. Let the end-graphs of $Y$ in $G$ be $H$ and $K$. We arrange the bridges of $Y$ in $M$ in two disjoint classes $T$ and $U$ so that those corresponding to separates of $H(K)$ are in $\boldsymbol{T}(\boldsymbol{U})$. If $Y$ is odd we may suppose that two distinct members $B_{1}$ and $B_{2}$ of $T$ overlap. Then $G \cdot B_{1}$ and $G \cdot B_{2}$ are separates of $H$. There are vertices $v_{1}$ of $G \cdot B_{1}$ and $v_{2}$ of $G \cdot B_{2}$ such that $G \cdot B_{2}$ is a subgraph of $C\left(B_{1}, v_{1}\right)$ and $G \cdot B_{1}$ is a subgraph of $C\left(B_{2}, v_{2}\right)$. It is clear that each vertex of $H$ is a vertex of $C\left(B_{1}, v_{1}\right)$ or $C\left(B_{2}, v_{2}\right)$. Hence $Y\left(B_{1}, v_{1}\right) \cup Y\left(B_{2}, v_{2}\right)$ $=Y$, contrary to the supposition that $B_{1}$ and $B_{2}$ overlap.

(8.3) The circuit-matroids of the Kuratowski graphs are not graphic.

Proof. For the complete 5-graph, as defined in $\$ 4$, we find by inspection that $\left(\left\{L_{13}\right\},\left\{L_{24}\right\},\left\{L_{35}\right\},\left\{L_{14}\right\},\left\{L_{25}\right\},\left\{L_{13}\right\}\right)$ is an odd overlap-circuit of the point $\left\{L_{12}, L_{23}, L_{34}, L_{45}, L_{15}\right\}$ of the circuit-matroid. For the Thomsen 
graph $\left(\left\{L_{12}\right\},\left\{L_{23}\right\},\left\{L_{31}\right\},\left\{L_{12}\right\}\right)$ is an odd overlap-circuit of the point $\left\{L_{11}, L_{21}, L_{22}, L_{32}, L_{33}, L_{13}\right\}$ of the circuit-matroid. (See Figure I.) Hence in each case the circuit-matroid is nongraphic, by (8.2).

(8.4) Let $Y$ be a point of a connected graphic matroid $M$ such that no two bridges of $Y$ in $M$ overlap. Then there exists a nonseparable graph $G$ such that $\boldsymbol{M}=\boldsymbol{B}(G)$ and $Y=Q(\{a\}, V(G)-\{a\})$ for some $a \in V(G)$.

Proof. There exists $G$ such that $\boldsymbol{M}=\boldsymbol{B}(G)$. We take $G$ to be nonseparable. This is possible by (4.11) since $\boldsymbol{M}=\boldsymbol{B}(G) \cdot E(G)=\boldsymbol{B}(G \cdot E(G)$ ) by (4.8). Let the end-graphs of $Y$ in $G$ be $G_{1}$ and $G_{2}$. We suppose $G, G_{1}$ and $G_{2}$ chosen so that $\alpha_{1}\left(G_{2}\right)$ has the least possible value.

Assume $\alpha_{1}\left(G_{2}\right)>0$. Then $\alpha_{1}\left(G_{1}\right)>0$. Choose separates $G \cdot B_{1}$ and $G \cdot B_{2}$ of $G_{1}$ and $G_{2}$ respectively. $B_{1}$ and $B_{2}$ are bridges of $Y$ in $M$, by (4.8) and (4.11). Since they do not overlap there are, by (8.1), vertices $v_{1}$ and $v_{2}$ of $G \cdot B_{1}$ and $G \cdot B_{2}$ respectively such that

$$
Y\left(B_{1}, v_{1}\right) \cup Y\left(B_{2}, v_{2}\right)=Y .
$$

Keeping $B_{2}$ and $v_{2}$ fixed we consider all possible choices of $B_{1}$ and $v_{1}$ for which (i) is true, and we select one for which $C\left(B_{1}, v_{1}\right)$ has the least possible number of edges.

Let $P_{1}, P_{2}, \cdots, P_{k}$, where $P_{1}=B_{1}$, be the sets of edges of the separates of $G_{1}$ having $v_{1}$ as a vertex. (See Figure II.) For each $P_{j}$ let $E_{j}$ be the subgraph of $G_{1}$ which is the union of $G \cdot P_{j}$ and those subgraphs $C\left(P_{j}, x\right)$ of $G_{1}$ such that $x \in V\left(G \cdot P_{j}\right)-\left\{v_{1}\right\}$. The graphs $E_{j}$ have the common vertex $v_{1}$. But by the definition of a separate no two of them have any other common vertex. Since $G_{1}$ is connected it is the union of the graphs $E_{j}$.

By hypothesis we can, for each $P_{j}$, find a vertex $p_{j}$ of $G \cdot P_{j}$ and a vertex $q_{j}$ of $G \cdot B_{2}$ such that

$$
Y\left(P_{j}, p_{j}\right) \cup Y\left(B_{2}, q_{j}\right)=Y .
$$

In accordance with (i) we take $p_{1}=v_{1}$ and $q_{1}=v_{2}$.

Since $G$ is nonseparable it follows from (ii) that for each $j$ there is an edge $a_{j}$ in $Y\left(B_{2}, q_{j}\right)$ but not in $Y\left(P_{j}, p_{j}\right)$ and an edge $b_{j}$ in $Y\left(P_{j}, p_{j}\right)$ but not in $Y\left(B_{2}, q_{j}\right)$.

Suppose $p_{j} \neq v_{1}$. Considering the edge $a_{1}$, of which one end is a vertex of $E_{1}$ other than $v_{1}$, we find that $q_{j}=q_{1}=v_{2}$, by (ii). But in this case $C\left(P_{j}, p_{j}\right)$ is a subgraph of $C\left(B_{1}, v_{1}\right)$ having fewer edges than $C\left(B_{1}, v_{1}\right)$. This contradicts the definition of $B_{1}$ and $v_{1}$. We deduce that $p_{j}=v_{1}$ for each $j$.

Considering the edge $a_{j}$ we see that $q_{j}$ is uniquely determined for each $P_{j}$. Let $Z_{j}$ denote the set of all members of $Y$ having one end a vertex of $E_{j}$ other than $v_{1}$. Then $Z_{j}$ is non-null since it includes $a_{j}$. By (ii) each member of $Z_{j}$ has its other end a vertex of $C\left(B_{2}, q_{j}\right)$.

If $x \in V\left(G \cdot B_{2}\right)$ we denote by $R(x)$ the subgraph of $G$ formed by taking the union of $C\left(B_{2}, x\right)$ and those graphs $E_{j}$ for which $q_{j}=x$, and then adjoining the members of the corresponding sets $Z_{j}$ as new edges. 


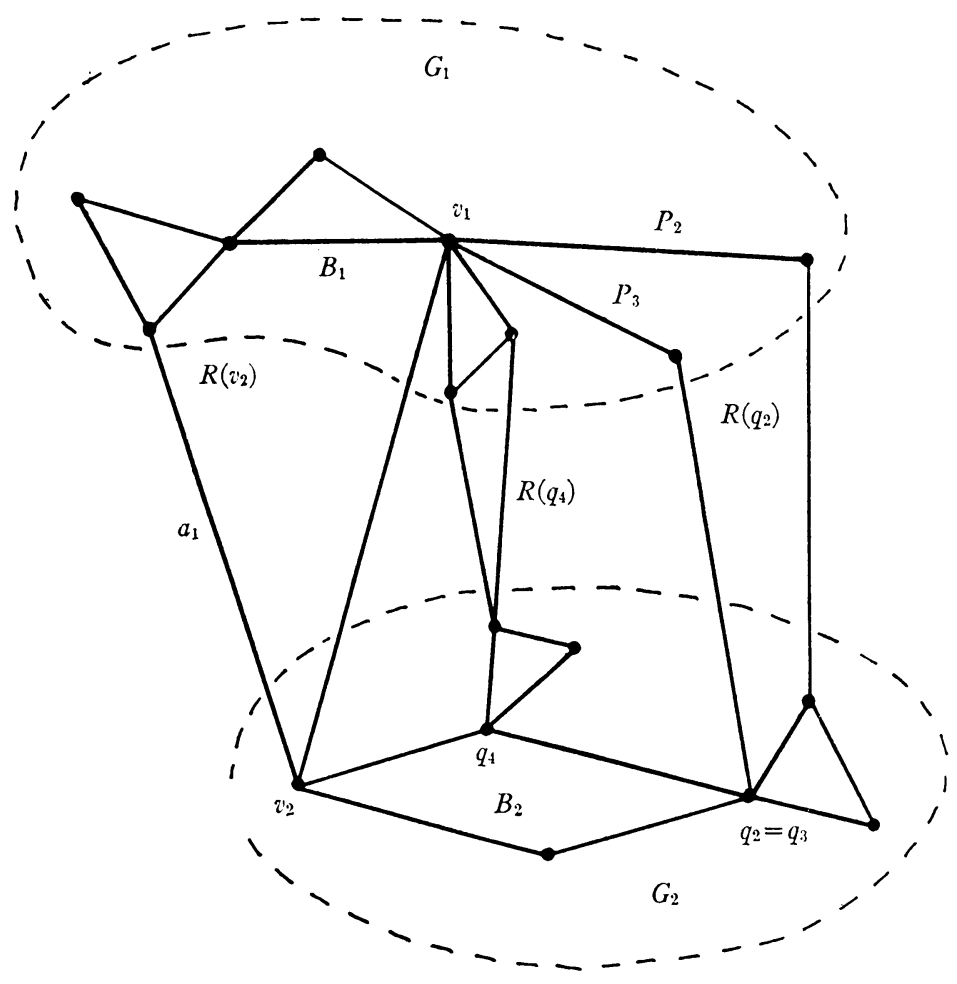

FIG. II

For a given $x$ the graph $R(x)$ may have only one vertex. If not the set $E(R(x))$ is non-null and its vertices of attachment in $G$ are $x$ and $v_{1}$. For since $G$ is nonseparable there are at least two such vertices of attachment, and $x$ and $v_{1}$ are the only possibilities. Moreover if $x$ and $y$ are distinct vertices of $G \cdot B_{2}$ then $R(x)$ and $R(y)$ have at most one common vertex $\left(v_{1}\right)$ and no comnon edge. We may therefore reverse all the non-null sets

$$
E(R(x)), x \in V\left(G \cdot B_{2}\right),
$$

without mutual inteference. By (4.12) $G$ is then transformed into another graph $G^{\prime}$ with the same bond-matroid $M$. Since $G^{\prime}$ has no isolated vertices we have $G^{\prime} \cdot E\left(G^{\prime}\right)=G^{\prime}$. Hence $G^{\prime}$ is nonseparable, by (4.11). But the transformation replaces $G_{1}$ by an end-graph $H$ of $Y$ in $G^{\prime}$ such that $E(H)=E\left(G_{2}\right)$ $-B_{2} \subset E\left(G_{2}\right)$. This is contrary to the choice of $G, G_{1}$ and $G_{2}$.

We deduce that $\alpha_{1}\left(G_{2}\right)=0$. The theorem follows.

For any binary matroid $M$ on a set $M$ there is a binary chain-group $N$ on $M$ such that $\boldsymbol{M}=\boldsymbol{M}(N)$. Any $\bmod 2$ sum $K$ of points of $\boldsymbol{M}$ must be the domain of a chain of $N$. Hence if $K$ is non-null it must have some point of $\boldsymbol{M}$ as a subset. We make use of this observation in the proof of the next two theorems. 
(8.5) Let $M$ be a connected regular matroid on a set $M$. Let $Y$ be an even point of $\boldsymbol{M}$ such that $\beta(\boldsymbol{M}, Y) \geqq 1$ and $\boldsymbol{M} \times(B \cup Y)$ is graphic for each bridge $B$ of $Y$ in $M$. Then $M$ is graphic.

Proof. If possible choose $M$ and $Y$ so that the theorem fails and $\alpha(M)$ has the least value consistent with this condition. Then $\beta(M, Y) \geqq 2$, since otherwise $\boldsymbol{M}=\boldsymbol{M} \times(B \cup Y)$, where $B$ is the only bridge of $Y$ in $\boldsymbol{M}$, and the theorem is trivially true.

Since $Y$ is even we can classify the bridges of $Y$ in $M$ in two disjoint classes $U_{1}$ and $U_{2}$ so that no two members of the same class overlap. Since $\beta(M, Y) \geqq 2$ we can arrange that $U_{1}$ and $U_{2}$ are both non-null. These conditions being satisfied we write $U_{1}$ and $U_{2}$ for the unions of the members of $U_{1}$ and $U_{2}$ respectively.

Let $B$ be any bridge of $Y$ in $M$. As $B$ is not a separator of $M$ it meets some point of $\boldsymbol{M} \cdot(M-Y)$. It must therefore be a connected flat of $\boldsymbol{M} \cdot(M-Y)$. The matroid $\boldsymbol{M} \times(B \cup Y)$ is connected. For otherwise we have $\boldsymbol{M} \cdot B$ $=(\boldsymbol{M} \cdot(M-Y)) \cdot B=(\boldsymbol{M} \cdot(M-Y)) \times B=\boldsymbol{M} \times B$, by (3.2), (3.9) and (6.3), contrary to the hypothesis that $M$ is connected. From this result it follows that $M \times\left(U_{i} \cup Y\right)$ is connected $(i=1,2)$. But $M \times\left(U_{i} \cup Y\right)$ is regular, by (3.7). Further the bridges of $Y$ in $\boldsymbol{M} \times\left(U_{i} \cup Y\right)$ are the members of $\boldsymbol{U}_{i}$ and no two of them overlap, by (7.4). Moreover for each $B \in U_{i}$ the matroid $\left(M \times\left(U_{i} \cup Y\right)\right) \times(B \cup Y)$ is identical with $\boldsymbol{M} \times(B \cup Y)$ and is therefore graphic. Hence $\boldsymbol{M} \times\left(U_{i} \cup Y\right)$ is graphic, by the choice of $\boldsymbol{M}$.

By (8.4) there exists a nonseparable graph $G_{i}$ and a vertex $w_{i}$ of $G_{i}$ such that $\boldsymbol{B}\left(G_{i}\right)=\boldsymbol{M} \times\left(U_{i} \cup Y\right)$ and such that $Y=Q\left(\left\{w_{i}\right\}, V\left(G_{i}\right)-\left\{w_{i}\right\}\right)$ in $G_{i}$.

We may take $V\left(G_{1}\right)$ and $V\left(G_{2}\right)$ to be disjoint subsets of some larger set $V$. We construct a graph $G^{\prime}$ as follows. $E\left(G^{\prime}\right)=M$ and $V\left(G^{\prime}\right)=\left(V\left(G_{1}\right) \cup V\left(G_{2}\right)\right)$ $-\left\{w_{1}, w_{2}\right\}$. If $A \in M-Y$ the ends of $A$ in $G^{\prime}$ are its ends in $G_{1}$ or $G_{2}$, but if $A \in Y$ its ends in $G^{\prime}$ are its end other than $w_{1}$ in $G_{1}$ and its end other than $w_{2}$ in $G_{2}$. (See Figure III.)

From this construction it follows that $G^{\prime}:(M-Y)$ has just two components $H_{1}$ and $H_{2}, H_{i}$ being the end-graph of $Y$ in $G_{i}$ not including $w_{i}$. Hence $Y \in \boldsymbol{B}\left(G^{\prime}\right)$. We note that $U_{i}$ is a separator of $\boldsymbol{B}\left(G^{\prime}\right) \cdot(M-Y)=\boldsymbol{B}\left(G^{\prime} \cdot(M-Y)\right)$ as well as of $\boldsymbol{M} \cdot(M-Y)$.

To change $G_{i}$ into $G^{\prime} \times\left(U_{i} \cup Y\right)$ we have only to replace $w_{i}$ by $H_{i}$ and each $v \in V\left(G_{i}\right)-\left\{w_{i}\right\}$ by the edgeless subgraph of $G^{\prime}$ having $v$ as its only vertex, arranging that corresponding vertices in the two graphs are incident with the same edges. We therefore have $\boldsymbol{B}\left(G^{\prime}\right) \times\left(U_{i} \cup Y\right)=\boldsymbol{B}\left(G^{\prime} \times\left(U_{i} \cup Y\right)\right)$ $=\boldsymbol{B}\left(G_{i}\right)=\boldsymbol{M} \times\left(U_{i} \cup Y\right)$, by (4.9). This implies that each $\boldsymbol{B}\left(G^{\prime}\right) \times\left(U_{i} \cup Y\right)$ is connected and hence that $B\left(G^{\prime}\right)$ is connected.

The mod 2 sum of the three points on any line of a binary matroid is null, by HII, (2.6). Hence the points of $\boldsymbol{B}\left(G^{\prime}\right)$ expressible as $\bmod 2$ sums of points of $\boldsymbol{M}$ constitute a convex subclass $\boldsymbol{C}$ of $\boldsymbol{B}\left(G^{\prime}\right)$. If $\boldsymbol{B}\left(G^{\prime}\right)-\boldsymbol{C}$ is not null there exists $X \in B\left(G^{\prime}\right)-\boldsymbol{C}$ such that $X \cup Y$ is a line of $B\left(G^{\prime}\right)$, by HI, (5.1). Then $X-(X \cap Y)$ is a point of $B\left(G^{\prime}\right) \cdot(M-Y)$, by (6.2). Since $U_{1}$ and $U_{2}$ are 

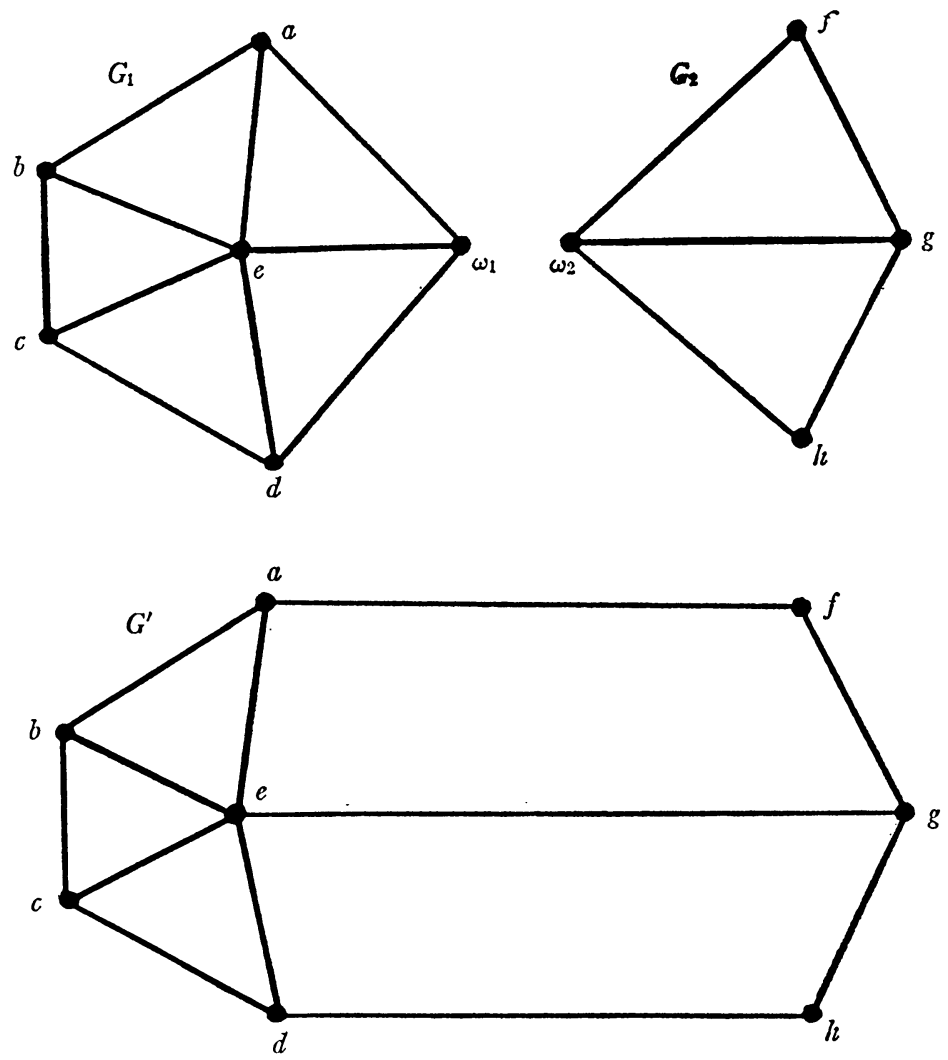

FIG. III

separators of this matroid we have $X \in B\left(G^{\prime}\right) \times\left(U_{j} \cup Y\right)$, where $j=1$ or 2 . But then $X \in M \times\left(U_{j} \cup Y\right) \subset M$, contrary to the definition of $X$.

We deduce that each point of $B\left(G^{\prime}\right)$ belongs to $C$. Hence for each $X \in B\left(G^{\prime}\right)$ there exists $X^{\prime} \in M$ such that $X^{\prime} \subseteq X$. A similar argument with $\boldsymbol{M}$ and $B\left(G^{\prime}\right)$ interchanged shows that for each $X^{\prime} \in M$ there exists $X \in B\left(G^{\prime}\right)$ such that $X \subseteq X^{\prime}$. Hence $\boldsymbol{M}=\boldsymbol{B}\left(G^{\prime}\right)$ by Axiom $\mathrm{I}$. This is impossible, by the choice of $M$. The theorem follows.

(8.6) Let $M$ be a regular matroid on a set $M$ such that $M$ is not graphic but all the other minors of $\boldsymbol{M}$ are graphic. Then $\boldsymbol{M}$ is the circuit-matroid of a Kuratowski graph.

Proof. Suppose $\boldsymbol{M}$ is not connected. We can find complementary non-null separators $S_{1}$ and $S_{2}$ of $\boldsymbol{M}$. There exist graphs $G_{1}$ and $G_{2}$ such that $B\left(G_{1}\right)$ $=\boldsymbol{M} \times S_{1}$ and $\boldsymbol{B}\left(G_{2}\right)=\boldsymbol{M} \times S_{2}$. We may take $V\left(G_{1}\right)$ and $V\left(G_{2}\right)$ to be disjoint subsets of some larger set $V$. Then $G_{1}$ and $G_{2}$ together constitute a graph $G$. We note that $S_{1}$ and $S_{2}$ are separators of $B(G)$.

If $X \in B(G)$ we have $X \in B(G) \times S_{i}=B(G) \cdot S_{i}=B\left(G \cdot S_{i}\right)=B\left(G_{i}\right)=M \times S_{i}$, where $i=1$ or 2 . Hence $X \in M$. We find similarly that if $X \in M$ then $X \in B(G)$. 
Hence $\boldsymbol{M}=\boldsymbol{B}(G)$, contrary to hypothesis.

We deduce that $\boldsymbol{M}$ is connected.

Suppose $d \boldsymbol{M}=-1$. Then $\alpha(M)=0$ or 1 since $\boldsymbol{M}$ is connected. We thus have $\boldsymbol{M}=\boldsymbol{B}(G)$ for some graph $G$ with just one vertex. If $\alpha(M)=0, G$ is edgeless; otherwise $E(G)$ consists of a single loop.

If $d \boldsymbol{M}=0$ we have $M \in M$. Then $\boldsymbol{M}=\boldsymbol{B}(G)$ where $G$ has just two vertices and each $A \in M$ is an edge of $G$ incident with both of them.

Suppose $d \boldsymbol{M}=1$. Then $M$ is a connected line of $\boldsymbol{M}$. Let its three points be $X_{1}, X_{2}$ and $X_{3}$. Each of these is the mod 2 sum of the others. Hence $X_{1} \cap X_{2}$ $\cap X_{3}=\varnothing$. But $X_{1} \cap X_{2}, X_{2} \cap X_{3}$ and $X_{3} \cap X_{1}$ are all non-null, by Axiom I, and their union is $M$. Let $G$ be a graph with just three vertices $v_{1}, v_{2}$ and $v_{3}$, and such that $E(G)=M$. We take each member of $X_{i} \cap X_{j}$ to be incident with $v_{i}$ and $v_{j}(1 \leqq i<j \leqq 3)$. It is readily verified that $\boldsymbol{M}=\boldsymbol{B}(G)$.

From the preceding analysis we conlude that $d M \geqq 2$.

Lemma I. Suppose $Y \in M$ and $\beta(M, Y) \geqq 2$. Then $Y$ is odd. Moreover the bridges of $Y$ in $M$ can be arranged in a single odd overlap-circuit $F(M, Y)$ so that two bridges overlap only if they are consecutive in $F(M, Y)$.

Proof. If $Y$ is even $M$ is graphic by (8.5), contrary to hypothesis. Let $F(\boldsymbol{M}, Y)$ be an odd overlap-circuit of $Y$ with as few terms as possible. Let the union of its terms be $S$. By (7.4) $Y$ is an odd point of $M \times S$. Hence $S=M$, by (8.2). Thus $F(\boldsymbol{M}, Y)$ includes all the bridges of $Y$ in $\boldsymbol{M}$. If any two bridges overlap and are not consecutive in $F(\boldsymbol{M}, Y)$ then $Y$, has an odd overlap-circuit with fewer terms than $F(\boldsymbol{M}, Y)$, which is impossible.

Lemma II. Let $Y$ be any odd point of $M$. Suppose some $Z \in M \cdot(M-Y)$ on a bridge $B$ of $Y$ in $\boldsymbol{M}$ is skew to both neighbours of $B$ in $F(\boldsymbol{M}, Y)$. Then $B$ is unicellular.

Proof. Write $S=M-(B-Z)$. Now $Y \cup Z$ is a line of $M \times S$. Hence $Z \in(M \times S) \cdot(S-Y)$, by (6.2). Moreover $Z$ determines the same partition of $Y$ in $\boldsymbol{M} \times S$ as in $\boldsymbol{M}$. Using (7.4) we deduce that $Y$ has an odd overlapcircuit in $\boldsymbol{M} \times S$ obtained from $F(\boldsymbol{M}, Y)$ by substituting $Z$ for $B$. Hence $S=M$, that is $B=Z$, by (8.2).

Let $Z$ determine the partition $\{U, V\}=\pi(M, Z, Y)$ of $Y$. Choose $a \in Z$ and write $T=M-(Z-\{a\})$. We have $Y \in \boldsymbol{M} \cdot T$, by (7.5). Now $\{a\}$ is a point of $(\boldsymbol{M} \cdot T) \cdot(T-Y)$ and a bridge of $Y$ in $\boldsymbol{M} \cdot T$. There exists $X^{\prime} \in M \cdot T$ such that $a \in X^{\prime} \subseteq Z \cup U \in M$, by HII, (3.1). There exists $X \in M$ such that $X \cap T=X^{\prime}$. By Axiom I, $X=Z \cup U$ and so $X^{\prime}=U \cup\{a\}$. Hence $\{a\}$ determines the same partition $\{U, V\}$ of $Y$ in $\boldsymbol{M} \cdot T$ as $Z$ does in $\boldsymbol{M}$. Using (7.5) we deduce that $Y$ has an odd overlap-circuit in $M \cdot T$ obtained from $F(M, Y)$ by substituting $\{a\}$ for $Z$. Hence $T=M$, that is $B=Z=\{a\}$, by (8.2).

Lemma III. If $Y \in M$ and $\beta(M, Y) \geqq 5$, then every bridge of $Y$ in $M$ is unicellular. 
Proof. By Lemma I $Y$ is odd. Let $B$ be any bridge of $Y$ in $M$ and let $B_{1}$ and $B_{2}$ be its two neighbours in $F(\boldsymbol{M}, Y)$. Now $B$ and $B_{1}$ are not equivalent 3-bridges, for otherwise $B_{1}$ and $B_{2}$ would overlap, contrary to Lemma I Hence $B$ is skew to $B_{1}$, and similarly to $B_{2}$, by (7.9). But $B_{1}$ and $B_{2}$ are not skew, by Lemma I. Lemma III now follows from (6.5) and Lemma II.

Choose $J \in M$ so that $\beta(M, J)$ has the greatest possible value and $\alpha(J)$ has the greatest value consistent with this. Since $d \boldsymbol{M} \geqq 2$ such a choice is possible and $\alpha(J)>1$ by the connection of $\boldsymbol{M}$. Moreover $\beta(\boldsymbol{M}, J) \geqq 2$, by (7.8). Hence $J$ is odd, by Lemma I. We can write

$$
F(\boldsymbol{M}, J)=\left(B_{1}, B_{2}, \cdots, B_{2 n \perp 1}, B_{1}\right),
$$

where $n$ is an integer $\geqq 1$. When convenient we write $B_{i}$ also as $B_{i+2 n+1}$.

Let $\pi$ be the class of all non-null sets of the form

$$
\bigcap_{i=1}^{2 n+1}\left(W_{i}\right),
$$

where $W_{i} \in \boldsymbol{\pi}\left(\boldsymbol{M}, B_{i}, J\right)$. Clearly the members of $\boldsymbol{\pi}$ are disjoint and their union is $J$.

Lemma IV. If $U \in \pi$, then $\alpha(U)=1$. Moreover there is an integer $i$ satisfying $1 \leqq i \leqq 2 n+1$ such that $J-U=W_{i} \cup W_{i+1}$ for some $W_{i} \in \pi\left(\boldsymbol{M}, B_{i}, J\right)$ and some $W_{i+1} \in \pi\left(\boldsymbol{M}, B_{i+1}, J\right)$.

Proof. Choose $a \in U$. Now $J-\{a\} \in M \cdot(M-\{a\})$, by (7.6). But $F(\boldsymbol{M}, J)$ is not an odd overlap-circuit of $J-\{a\}$ in $\boldsymbol{M} \cdot(M-\{a\})$, by (8.2). Hence $U=\{a\}$, by (7.6). Further we can find an integer $i$ satisfying $1 \leqq i \leqq 2 n+1$ such that $B_{i}$ and $B_{i+1}$ do not overlap as bridges of $J-\{a\}$ in $\boldsymbol{M} \cdot(M-\{a\})$. The lemma follows.

Lemma V. If $1 \leqq i \leqq 2 n+1$ there is no $W \in \pi\left(M, B_{i}, J\right)$ such that $W \in \boldsymbol{\pi}$.

Proof. Suppose such a $W$ exists. By (7.3) there exists $Z \in \boldsymbol{M} \cdot(M-J)$ on $B_{i}$ such that $W \cup Z \in M \times\left(B_{i} \cup J\right)$. If $\alpha(Z)=1$ we must have $B_{i}=Z$. Then $\boldsymbol{\pi}\left(\boldsymbol{M}, B_{i}, J\right)$ is the partition $\{W, J-W\}$ of $J$ determined by $Z$. This is impossible, by Lemma I, since it implies that $B_{i}$ overlaps none of the other bridges.

If $\alpha(Z)>1$ we write $J^{\prime}=(J-W) \cup Z$. Then all the bridges of $J$ in $\boldsymbol{M}$ other than $B_{i}$ are bridges of $J^{\prime}$ in $M$, by (7.7), and there is at least one other bridge of $J^{\prime}$, meeting $W$, in $\boldsymbol{M}$. But this implies $\beta\left(\boldsymbol{M}, J^{\prime}\right) \geqq \beta(\boldsymbol{M}, J)$ and $\alpha\left(J^{\prime}\right)>\alpha(J)$, contrary to the definition of $J$. The lemma follows.

Suppose $n \geqq 2$. Then each bridge of $J$ in $M$ is unicellular, by Lemma III. Hence, for each $i, B_{i}$ is a point of $\boldsymbol{M} \cdot(M-J)$ determining a partition $\left\{S_{i}, T_{i}\right\}$ of $J$. Moreover $S_{i}$ and $T_{i}$ are both non-null, since consecutive members of 
$F(\boldsymbol{M}, J)$ overlap. Clearly $\boldsymbol{\pi}\left(\boldsymbol{M}, B_{i}, J\right)=\left\{S_{i}, T_{i}\right\}$. Consecutive members of $F(M, J)$ are skew, by (7.9). We write $S_{i}=S_{i+2 n+1}, T_{i}=T_{i+2 n+1}$.

Consider a particular bridge $B_{i}$. By Lemma I we can adjust the notation so that $S_{j} \subset S_{i}$ or $S_{j} \subset T_{i}$ whenever $B_{j}$ is distinct from and not consecutive with $B_{i}$ in $F(M, J)$. We choose one $B_{j}$ of this kind and arrange, by interchanging $S_{i}$ and $T_{i}$ if necessary, that $S_{j} \subset T_{i}$. Let $B_{k}$ be the first member of the sequence $\left(B_{j}, \cdots, B_{i+2 n-1}\right)$ such that $S_{k+1} \cap S_{i} \neq \varnothing$. Then $S_{k+1}$ meets both $S_{i}$ and $T_{i}$, since $B_{k+1}$ is skew to $B_{k}$. Hence $B_{k+1}$ is consecutive with $B_{i}$ in $F(\boldsymbol{M}, J)$, that is $k=i+2 n-1$. Similarly if $B_{l}$ is the last member of the sequence $\left(B_{i+2}, \cdots, B_{j}\right)$ such that $S_{l-1} \cap S_{i} \neq \varnothing$ we find that $l=i+2$. It follows that $S_{h} \subset T_{i}$ for each $B_{h}$ not consecutive with $B_{i}$ in $F(\boldsymbol{M}, J),(h \neq i)$.

By the result just proved we can adjust the notation so that $S_{i}$ contains neither $S_{j}$ nor $T_{j}$ as a subset whenever $B_{i}$ and $B_{j}$ are distinct. Then, by Lemma I, $S_{i} \cap S_{j}$ is non-null if and only if $B_{i}$ and $B_{j}$ are consecutive in $F(M, J)$.

It follows that the $2 n+1$ sets $S_{i} \cap S_{i+1}(1 \leqq i \leqq 2 n+1)$ are distinct members of $\boldsymbol{\pi}$. We proceed to show that they are the only members of $\boldsymbol{\pi}$. Choose any $W \in \pi$. By Lemma IV we can write $W=S_{i} \cap S_{i+1}, S_{i} \cap T_{i+1}, T_{i} \cap S_{i+1}$ or $T_{i} \cap T_{i+1}$, for some $i$ satisfying $1 \leqq i \leqq 2 n+1$. In the first case there is nothing to prove. In the second case we observe that $S_{i-1}$ (or $S_{i+2 n}$ ) meets $S_{i}$ and is a subset of $T_{i+1}$. Hence $W$ is the member $S_{i-1} \cap S_{i}$ (or $S_{i+2 n} \cap S_{i+2 n+1}$ ) of $\pi$. We dispose of the third case in a similar way, using $S_{i+2}$ instead of $S_{i-1}$. In the fourth case we have $S_{i+3} \subseteq T_{i} \cap T_{i+1}=W$, whence $S_{i+3}=W$ and Lemma V is contradicted.

If $n>2$ we write $J^{\prime}=\left(J-S_{1}\right) \cup B_{1}$. Then, by (7.7) $J^{\prime} \in M$ and $B_{3}, B_{4}, \cdots$, $B_{2 n}$ are bridges of $J^{\prime}$ in $\boldsymbol{M}$. By Lemma $\mathrm{I}, J^{\prime}$ is odd and so $\beta\left(\boldsymbol{M}, J^{\prime}\right) \geqq 5$. Hence $\beta\left(\boldsymbol{M}, J^{\prime}\right)=\alpha(M)-\alpha\left(J^{\prime}\right)$, by Lemma III. But $\alpha\left(J^{\prime}\right)=\alpha(J)-\alpha\left(S_{2 n+1} \cap S_{1}\right)$ $-\alpha\left(S_{1} \cap S_{2}\right)+1<\alpha(J)$. Hence $\beta\left(\boldsymbol{M}, J^{\prime}\right)>\alpha(M)-\alpha(J)=\beta(\boldsymbol{M}, J)$, contrary to the definition of $J$.

If $n=2$ we write $S_{i} \cap S_{i+1}=\left\{L_{i, i+1}\right\}$ if $1 \leqq i \leqq 4$, and $S_{5} \cap S_{1}=\left\{L_{15}\right\}$ $\left(\alpha\left(S_{i} \cap S_{i+1}\right)=1\right.$ by Lemma IV). We also write $B_{i}=\left\{L_{i, i+2}\right\}$ if $1 \leqq i \leqq 3, B_{4}$ $=\left\{L_{14}\right\}$ and $B_{5}=\left\{L_{25}\right\}$. We construct a graph $G$ such that $E(G)=M$. We take $G$ to have just five vertices $v_{1}, v_{2}, v_{3}, v_{4}$ and $v_{5}$, the ends of $L_{i j}$ in $G$ being $v_{i}$ and $v_{j}$. Then $G$ is a complete 5 -graph and the notation is that used in $\S 4$. We find that $\boldsymbol{M}=\boldsymbol{C}(G)$. To prove this we observe that $J$ and the five sets $B_{i} \cup S_{i}$ are points of both $\boldsymbol{M}$ and $\boldsymbol{C}(G)$. Any point $X$ of $\boldsymbol{M}$ is a linear combination mod 2 of these six. For otherwise some linear combination of points of $M$ would be a non-null proper subset of $J$ and would have a point of the binary matroid $M$ as a subset, contrary to Axiom I. Hence $X$ is a mod 2 sum of points of $\mathbf{C}(G)$ and so has a point of the binary matroid $\mathbf{C}(G)$ as a subset. Similarly each point of $\boldsymbol{C}(G)$ has a point of $\boldsymbol{M}$ as a subset. Hence $\boldsymbol{M}$ is $\boldsymbol{C}(G)$, the circuit-matroid of a Kuratowski graph, by Axiom I. 
In the remaining case $n=1$ and we can write $F(\boldsymbol{M}, J)=\left(B_{1}, B_{2}, B_{3}\right)$. By Lemma $\mathrm{V}, B_{1}, B_{2}$ and $B_{3}$ are not mutually equivalent 3-bridges.

Suppose $\boldsymbol{\pi}\left(\boldsymbol{M}, B_{1}, J\right)=\boldsymbol{\pi}\left(\boldsymbol{M}, B_{2}, J\right)=\left\{W_{1}, W_{2}, W_{3}\right\}$. Then $B_{3}$ is skew to $B_{1}$ and $B_{2}$, by (7.9). But $B_{1}$ and $B_{2}$ are clearly not skew. Hence $B_{3}$ is unicellular, by (6.5) and Lemma II. Let it determine the partition $\{S, T\}$ of $J$. We may suppose without loss of generality that $W_{1} \cap S$ is non-null. Then $W_{1} \cap S \in \boldsymbol{\pi}$. It is a proper subset of $W_{1}$, by Lemma V. By Lemma IV we may write, without loss of generality, $J-\left(W_{1} \cap S\right)=T \cup W_{i}$, where $i=1$ or 2 . (If $J-\left(W_{1} \cap S\right)=S \cup W_{i}$ then $J=S \cup W_{i}$, which is impossible since $B_{1}$ and $B_{3}$ overlap.) But then $W_{3} \subseteq T, W_{3} \in \pi$, and Lemma $\mathrm{V}$ is contradicted.

By (7.9) we may now suppose that $B_{1}, B_{2}$ and $B_{3}$ are mutually skew. Applying (6.5) and Lemma II we find that two of them, say $B_{1}$ and $B_{2}$ are unicellular. Let them determine partitions $\left\{S_{1}, T_{1}\right\}$ and $\left\{S_{2}, T_{2}\right\}$ respectively of $J$.

Assume $\boldsymbol{\pi}\left(\boldsymbol{M}, B_{3}, J\right)=\left\{W_{1}, W_{2}, \cdots, W_{k}\right\}$, where $k \geqq 3$.

Suppose $S_{1} \cap S_{2}$ is not a subset of any $W_{i}$. Then without loss of generality we may assume it meets both $W_{1}$ and $W_{2}$. But neither $W_{1}$ nor $W_{2}$ is a subset of $S_{1} \cap S_{2}$, by Lemma V. By Lemma IV and the overlapping of the three bridges we have either

$$
J-\left(S_{1} \cap S_{2} \cap W_{1}\right)=T_{1} \cup T_{2}
$$

or

$$
J-\left(S_{1} \cap S_{2} \cap W_{1}\right)=T_{x} \cup W_{u},
$$

where $x=1$ or 2 and $2 \leqq u \leqq k$. The first alternative must be ruled out since it implies $S_{1} \cap S_{2} \cap W_{2}=\varnothing$. Adopting the second we see that $W_{1}$ and $W_{u}$ are the only members of $\boldsymbol{\pi}\left(\boldsymbol{M}, B_{3}, J\right)$ meeting $S_{1} \cap S_{2}$. Hence $W_{u}=W_{2}$. A similar argument, with $W_{2}$ replacing $W_{1}$, gives

$$
J-\left(S_{1} \cap S_{2} \cap W_{2}\right)=T_{y} \cup W_{1},
$$

where $y=1$ or 2 . We have $x \neq y$ since otherwise $S_{3-x} \cup T_{x}=J$ and $B_{1}$ is not skew to $B_{2}$. But this implies $W_{3} \subseteq T_{1} \cap T_{2}$, which contradicts Lemma V.

We deduce that $S_{1} \cap S_{2} \subseteq W_{i}$ for some $i$. Similarly each of the sets $S_{1} \cap T_{2}$, $T_{1} \cap S_{2}$ and $T_{1} \cap T_{2}$ is a subset of some member of $\pi\left(\boldsymbol{M}, B_{3}, J\right)$. Hence, since $k \geqq 3$, we can adjust the notation so that $S_{1} \cap S_{2}=W_{1}$, which is contrary to Lemma V.

The above argument shows that $B_{1}, B_{2}$ and $B_{3}$ are all 2-bridges. They are all unicellular, by Lemma II and (7.9). We write $\left\{S_{3}, T_{3}\right\}$ for the partition of $J$ determined by $B_{3}$.

Now $S_{3}$ meets either both $S_{1} \cap S_{2}$ and $T_{1} \cap T_{2}$ or both $S_{1} \cap T_{2}$ and $T_{1} \cap S_{2}$. For otherwise $S_{3}$ would not meet all the sets $S_{1}, S_{2}, T_{1}$ and $T_{2}$, which is impossible since $B_{1}, B_{2}$ and $B_{3}$ are mutually skew by (7.9). Without loss of generality we suppose $S$ meets both $S_{1} \cap S_{2}$ and $T_{1} \cap T_{2}$. We may also assume 
that $S_{1} \cap T_{2} \subseteq S_{3}$, by (7.2). Hence $T_{3}$ meets both $S_{1} \cap S_{2}$ and $T_{1} \cap T_{2}$, since $B_{3}$ is skew to $B_{1}$ and $B_{2}$, and $S_{2} \cap T_{1} \subseteq T_{3}$, by (7.2).

Using Lemma IV we write $S_{1} \cap S_{2} \cap S_{3}=\left\{L_{11}\right\}, S_{1} \cap T_{2} \cap S_{3}=\left\{L_{21}\right\}$, $T_{1} \cap T_{2} \cap S_{3}=\left\{L_{22}\right\}, T_{1} \cap T_{2} \cap T_{3}=\left\{L_{32}\right\}, T_{1} \cap S_{2} \cap T_{3}=\left\{L_{33}\right\}$ and $S_{1} \cap S_{2} \cap T_{3}$ $=\left\{L_{13}\right\}$. We write also $B_{1}=\left\{L_{23}\right\}, B_{2}=\left\{L_{31}\right\}$ and $B_{3}=\left\{L_{12}\right\}$. We construct a graph $G$ such that $E(G)=M$. We take $G$ to have just six vertices $a_{1}, a_{2}, a_{3}$, $b_{1}, b_{2}, b_{3}$, the ends of $L_{i j}$ being $a_{i}$ and $b_{j}$. Then $G$ is a Thomsen graph and the notation is that used in $\$ 4$. By an argument like that already used for the complete 5 -graph we find $\boldsymbol{M}=\boldsymbol{C}(G)$. Thus $\boldsymbol{M}$ is the circuit-matroid of a Kuratowski graph, and the proof of (8.6) is complete.

We establish the main theorem as follows. If $\boldsymbol{M}$ is a graphic matroid it is regular, by (4.1), and its minors are all graphic, by (4.10). Hence it has no minor which is the circuit-matroid of a Kuratowski graph, by (8.3). Conversely suppose $\boldsymbol{M}$ is a regular matroid having no such minor. Then either $\boldsymbol{M}$ is graphic or it has a non-graphic minor $\boldsymbol{M}_{0}$ whose minors other than itself are all graphic, by (3.5). But if the second alternative holds $\boldsymbol{M}_{0}$ is the circuitmatroid of a Kuratowski graph, by (3.7) and (8.6).

To complete the proof we prove the dual of the foregoing result by applying (2.12), (3.6) and (4.2).

9. Figures and minors. The main theorems of HI and HII deal with geometrical figures whereas the main theorem of the present paper is expressed in terms of minors. We now show the relationship between the two concepts.

By a figure in a matroid $M$ we mean any collection of flats of $M$ which with $S$ and $T$ includes also $\langle S \cap T\rangle$. The figure of $M$ is the collection of all flats of $\boldsymbol{M}$. Two figures, possibly in different matroids, are equivalent if there is a 1-1 mapping (equivalence) of one onto the other which preserves dimension and inclusion relations (both ways).

Two cells of a matroid $\boldsymbol{M}$ are equivalent if no $X \in M$ contains one but not the other.

Two matroids $M$ and $\boldsymbol{M}^{\prime}$, on sets $M$ and $M^{\prime}$ respectively, are isomorphic if there is a 1-1 mapping $f$ of $M$ onto $M^{\prime}$ which maps the points of $M$ onto the points of $\boldsymbol{M}^{\prime}$.

(9.1) Let $\boldsymbol{M}$ and $\boldsymbol{M}_{0}$ be matroids on sets $M$ and $M_{0}$ respectively, $\boldsymbol{M}_{0}$ being connected and having no two cells equivalent. Then $M$ has a figure equivalent to that of $\boldsymbol{M}_{0}$ if and only if it has a minor isomorphic with $\boldsymbol{M}_{0}$.

Proof. Suppose the minor $(\boldsymbol{M} \times S) \cdot T$ of $\boldsymbol{M}$ is isomorphic with $\boldsymbol{M}_{\mathbf{0}}$. We apply the theory of HII, $\S 3$, as follows. The minor can be written as $(\boldsymbol{M} \times Z)$ $\cdot T$, where $Z$ is a carrier of $(M \times S) \cdot T$ in $M \times S$. The figure of $(M \times Z) \cdot T$ is equivalent to that of $M_{0}$, and the $(T, Z)$-mapping of $\boldsymbol{M} \times S$ maps it onto an equivalent figure in $\boldsymbol{M} \times Z$, and therefore in $\boldsymbol{M}$.

Conversely suppose $\boldsymbol{M}$ has a figure $F$ equivalent to that of $\boldsymbol{M}_{0}$ under an equivalence $f$. We may assume $\boldsymbol{M}_{0}$ is not a null class since otherwise the theorem is trivially true. Hence there exists $S \in F$ such that $f(S)=M_{0}$, and 
$d=d S \geqq 0$. Enumerate the $(d-1)$-flats of $F$ as $U_{1}, \cdots, U_{k}$. For each $U_{i}$ we can find $a_{i} \in S$ such that $U_{i}=\left\langle S-\left\{a_{i}\right\}\right\rangle$, by HI, $\$ 2$. We can write the $(d-1)$ flat $f\left(U_{i}\right)$ of $M_{0}$ as $\left\langle M_{0}-\left\{b_{i}\right\}\right\rangle$ for some $b_{i} \in M_{0}$. Let $T$ be the set of the $k$ cells $a_{i}$. Since $\boldsymbol{M}_{0}$ has no two equivalent cells there is a 1-1 mapping $g$, defined by $g\left(a_{i}\right)=b_{i}$, of $T$ onto $M_{0}$.

If $U \in F$, then $U \cap T$ is a flat of $(M \times S) \cdot T$, by HI, (3.1). Conversely if $W$ is any flat of $(M \times S) \cdot T$ let $W^{\prime}$ be the union of the points of $M \times S$ common to all $\left\langle S-\left\{a_{i}\right\}\right\rangle$ such that $a_{i} \in T-W$. Then $W^{\prime} \in F$. It is clear that two distinct flats of $F$ cannot have the same intersection with $T$. For by the equivalence of $F$ and the figure of $M_{0}$ they can all (except $S$ ) be expressed as geometrical intersections of $(d-1)$-flats of $F$. Hence the operation of taking intersections with $T$ defines an equivalence of $F$ onto the figure of $(\boldsymbol{M} \times S)$ - T. Accordingly $g$ defines an isomorphism of $(M \times S) \cdot T$ and $M_{0}$.

We can use this result to express the main theorem (4.5) of HII in terms of minors. For in HII, $\$ 4$, constructions are given for matroids whose figures are of Type BI or BII, and these matroids are connected and have no two cells equivalent. On the other hand we can state the main theorem of the present paper as follows: a matroid $M$ is graphic (cographic) if and only if it is regular and has no figure equivalent to that of the circuit-matroid (bondmatroid) of a Kuratowski graph.

\section{REFERENCES}

1. C. Kuratowski, Sur le probleme des courbes gauches en topologie, Fund. Math. vol. 15 (1930) pp. 305-331.

2. W. T. Tutte, A class of abelian groups, Canad. J. Math. vol. 8 (1956) pp. 13-28.

3. - A homotopy theorem for matroids, I, Trans. Amer. Math. Soc. vol. 88 (1958) pp. 144-160.

4. - A homotopy theorem for matroids, II, Trans. Amer. Math. Soc. vol. 88 (1958) pp. 161-174.

5. H. Whitney, Nonseparable and planar graphs, Trans. Amer. Math. Soc. vol. 34 (1932) pp. 339-362. 507-533.

6. - The abstract properties of linear dependence, Amer. J. Math. vol. 57 (1935) pp.

UNIVERSITY OF TORONTO

Toronto, Ont. 